\title{
Hydro-mechanical response of collapsible soils under different infiltration events
}

\author{
Rotisciani G.M. ${ }^{1}$, Sciarra G. ${ }^{2}$, Casini F. ${ }^{3}$, Desideri A. ${ }^{1}$ \\ ${ }^{1}$ Dipartimento Ingegneria Strutturale e Geotecnica, \\ Università di Roma La Sapienza, Via Eudossiana 18, 00184 Rome, Italy \\ giada.rotisciani@uniroma1.it,augusto.desideri@uniroma1.it \\ 2 Dipartimento Ingegneria Chimica Materiali Ambiente, \\ Università di Roma La Sapienza, Via Eudossiana 18, 00184 Rome, Italy \\ giulio.sciarra@uniroma1.it \\ ${ }^{3}$ Dipartimento di Ingegneria Civile e Ingegneria Informatica \\ Università di Roma Tor Vergata, Via Politecnico 1, 00133 Rome, Italy \\ francesca.casini@uniroma2.it
}

\begin{abstract}
The behavior of a partially saturated soil during surface water infiltration is analysed by means of an elasto-plastic constitutive model formulated in terms of effective-stress and extended to unsaturated conditions. The model is calibrated considering laboratory scale experimental results under suction controlled conditions. The wetting process in two collapsing soils, initially loaded at in-situ stresses, is simulated by imposing two different boundary conditions: surface ponding and water flow. The stress paths resulting from the imbibition process are analyzed at different points inside the layer.
\end{abstract}

Keywords: Water Infiltration, Volumetric Collapse, Stress Paths, Partial Saturation, Constitutive Modeling.

\section{Introduction}

The prediction of the hydro-mechanical response of geomaterials, under rainfall events, plays a fundamental role in order to prevent phenomena such as foundation settlements or slope instability. Unsaturated soils during an imbibition path may either swell or shrink depending on the initial conditions in terms of void ratio and water content. The paper is focused on the analysis of the hydro-mechanical response of two collapsible soils subject to two different infiltration events with the aim of understanding how wetting-induced deformations affect the response of natural deposits during imbibition. Examples of the effects of variations of the saturation degree induced by wetting on the soil deformation 
can be found in [1] and [2], at laboratory scale, or in [3] and [4], at the field scale; moreover the effects of wetting on strength reduction are discussed in [5], [6] and [7].

A simple analysis of the water infiltration process shows that the current spatial distribution of the pore-water pressure is affected, on one hand, by the hydraulic properties of the soil, say retention characteristics and permeability, and, on the other hand, by the external loading due to climate conditions (rainfall intensity and duration, rainfall patterns and evapo-transpiration rate). The effects of these factors on the water infiltration and the response of the material in terms of pore-water pressure distribution have been investigated among others by [8], who developed a parametric study of rainfall infiltration mechanisms, or, more recently, by [9] and [10] who discussed the relation between the stability of shallow landslides and rainfall events for volcanic ashes and shallow weathered soils. However, in order to fully understand these phenomena, a consistent model of the hydro-mechanical coupling between water and solid grains must be taken into account. During soil imbibition, changes in the pore-water pressure distribution are induced by variations of the degree of saturation, which modify in turn the hydraulic regime of the soil and induce deformations of the solid matrix. Conversely, any change in the mechanical loading can influence the saturation process. It is indeed the hydro-mechanical coupled response of the material that is responsible of the most common instabilities caused by water infiltration: soil failure and excessive settlements, under collapse or shear strength reduction, and the corresponding damage to adjacent structures.

Different hydro-mechanical constitutive models have been developed in the last decades, starting from the pioneering paper in which the Barcelona Basic Model (BBM) has been formulated [11]. Adopting the same framework as that of BBM, several authors provide a characterization of the stress state in terms of net stress and suction, see e.g. [12], [13] and [14], and references therein. Alternatively, models have been developed on the basis on the concept of Bishop's effective stress and suction, see for a review [15] and [16], or some recent contribution as [17], [18, 19], [20], [21], [22], [23], [24] and [25]. In particular, the Modified Cam-Clay model adopted in this work has been extended to unsaturated conditions following this last approach, see [26] and [27]. It accounts for the elasto-plastic behavior of the soil and reduces to the Modified Cam-Clay model when saturation is attained. The mechanism of water storage is described by relating suction to a measure of water volume (volumetric or gravimetric water content, degree of saturation). In the most general case, this relation is hysteretic, stress path dependent and affected by the pore network distribution, see e.g.[28], [29], [30], [31], [32], [33], [34]. A possibility to explicit this relationship is to use an improved expression of Van Genuchten type, where hysteresis and dependency on void ratio or plastic strain are introduced. Some authors have given thermo-mechanical bases for such relationships, where hysteresis is associated to non-vanishing dissipation and strain dependency to the locked part of the energy (see for example [35]). In this paper, we discuss the general framework to model these different features and further used to formulate a hydro-mechanical model for collapsible materials.

This model is used to study the coupled response of an horizontal layer of partially saturated soil under wetting conditions, with the objective of analyzing and describing the consequences of two rainfall events. The parameters of the layer have been calibrated on two real soils: a volcanic ash, taken from the site of Cervinara, near Naples (Italy), see [36] and a clayey silt from Jossigny, near Paris (France), see [37]. The response of the soils to two different infiltration events has been studied: a moderate rainfall with intensity reaching the 0.4 of the saturated permeability and an intense rainfall modelled 
with zero pore-water pressure at the ground surface. The two scenarios lead to different hydraulic and mechanical responses in terms of stress paths, degree of saturation, collapse plastic strains and displacements at the ground level. A comparison between the results obtained for the two materials is presented for two reference points at different depths together with the profiles along the depth for significant dimensionless times.

The paper is organized as follows. In section 1 the details of the poromechanical model are provided deducing the constitutive laws from thermodynamics. In section 2 we illustrate the calibration of the constitutive model with respect to laboratory tests. In sections 3 and 4 we numerically simulate the wetting process of the layer, which is initially normally consolidated and subjected to the aforementioned boundary conditions at the ground surface. A comparison between the results obtained for the two materials is presented.

\section{The poromechanical model: thermodynamical restric- tions and constitutive laws}

A poromechanical model is adopted for describing the porous skeleton through which a partly saturating fluid can flow. Let $\mathcal{V}_{s}$ be the velocity of the material particles which constitute the skeleton and $\varepsilon_{i j}$ the strain field; the pore space is occupied by a wetting liquid $(w)$ and a non-wetting phase, constituted in particular by a gaseous mixture of vapor and dry air $(a)$. The liquid is assumed incompressible and the effect of the partial saturation is described by introducing an order parameter, say the degree of saturation $S_{r}$ of the wetting phase into the current pore space. The apparent densities of the wetting fluid and the gas, with respect to the unit volume of the porous medium, are prescribed by the product of the intrinsic densities, $\rho_{w}$ and $\rho_{a}$, the degree of saturation of the two phases, $S_{w}=S_{r}$ and $S_{a}:=1-S_{r}$, and the Eulerian porosity $(n)$; moreover they are assumed to satisfy the corresponding mass balance equations. Within this framework the Lagrangian continuity equation relative to fluid $\pi=w, a$, in the reference configuration of the solid skeleton, is

$$
\frac{d m_{\pi}}{d t}+\mathrm{M}_{k, k}^{\pi}=0
$$

where $m_{\pi}:=\rho_{\pi} \phi S_{\pi}$ is the fluid mass content related to fluid $\pi, \phi$ is the Lagrangian porosity and $\mathrm{M}_{k}^{\pi}:=\rho_{\pi} \phi S_{\pi}\left(\mathcal{V}_{k}^{\pi}-\mathcal{V}_{k}^{s}\right)$ indicates the $k$-th component of the Lagrangian flow of the $\pi$-th phase, through the solid if only small deformations of the skeleton are taken into account. In the following the usually named seapage velocity is defined as $\mathrm{v}_{k}:=\mathcal{V}_{k}^{w}-\mathcal{V}_{k}^{s}$. For more details we refer to [38] and [39].

Once recalled kinematics, the poromechanical constitutive model, capable for describing the behavior of partially saturated soils will be deduced extending classical thermodynamical arguments. We remind that the Clausius-Duhem inequality for a porous medium, saturated by a liquid, under isothermal conditions and purely gravitational body forces reads as, see [38],

$$
\sigma_{i j} \dot{\varepsilon}_{i j}+p_{w} \frac{d \phi}{d t}-\left(\frac{1}{\rho_{f}} p_{w, k}-g_{k}\right) \mathrm{M}_{k}-\frac{d \Psi_{s}}{d t} \geq 0
$$

where $\mathrm{M}_{k}$ in this case represents the Lagrangian flow vector relative to the saturating fluid, $\sigma_{i j}$ is the $(i, j)$-th component of the stress tensor acting on the overall porous medium, $p_{w}$ 
the pore-fluid pressure, $g_{k}$ the $k$-th component of the gravitational acceleration and $\dot{\phi}$ the derivative of the Lagrangian porosity, which in the case of small deformations, linearly depends on the variations of porosity and volumetric strain (positive in compression), say $\dot{\phi}=\dot{n}-n_{0} \dot{\varepsilon}_{v}$. Finally $\Psi_{s}$ is the Helmoltz free energy of the solid skeleton. Assuming the incompressibility of the solid grains implies the volumetric strain to be $\varepsilon_{v}=-\Delta n /(1-$ $\left.n_{0}\right)$. In this case Terzaghi's effective stress $\sigma_{i j}^{\prime}$ tensor naturally arises in the dissipation inequality:

$$
\sigma_{i j}^{\prime} \dot{\varepsilon}_{i j}-\left(\frac{1}{\rho_{f}} p_{w, k}-g_{k}\right) \mathrm{M}_{k}-\frac{d \Psi_{s}}{d t} \geq 0, \quad \sigma_{i j}^{\prime}:=-\left(p-p_{w}\right) \delta_{i j}+\operatorname{dev} \sigma_{i j} ;
$$

where the mean stress $p$ is conventionally assumed positive in compression and $\operatorname{dev} \sigma_{i j}$ is the $(i, j)$-th component of the deviatoric stress. If dissipation is split into two different contributions, one relative to the solid and one to the fluid, the classical constitutive laws for Terzaghi's effective stress as well as standard Darcy's law can be retrieved, once the solid dissipation vanishes and the fluid one is prescribed as a quadratic function of the fluid flow vector $\mathrm{M}_{k}$.

Following now [40] and [41], the dissipation inequality (2) can be extended to the case of unsaturated porous media, considering the contributions of a wetting fluid and a non-wetting mixture of vapor and air:

$$
\sigma_{i j} \dot{\varepsilon}_{i j}+p_{a} \frac{d \phi_{a}}{d t}+p_{w} \frac{d \phi_{w}}{d t}-\left(\frac{1}{\rho_{a}} p_{a, k}-g_{k}\right) \mathbf{M}_{k}^{a}-\left(\frac{1}{\rho_{w}} p_{w, k}-g_{k}\right) \mathbf{M}_{k}^{w}-\frac{d \Psi_{s}}{d t} \geq 0,
$$

where $\phi_{a}=\phi S_{a}=\phi\left(1-S_{r}\right)$ and $\phi_{w}=\phi S_{r}$ are the Lagrangian porosities of the two saturating fluids. In equation (4) we a-priori neglected the molecular diffusion of the vapor through the gas and treat the vapor-air mixture as an overall constitutent which can only be trasported by Darcean advection through the pores; in other words no Fickian contribution to dissipation is taken into account.

Assuming incompressibility of the solid grains implies Bishop's stress tensor

$$
\sigma_{i j}^{\prime}:=\left(p-\left(1-S_{r}\right) p_{a}-S_{r} p_{w}\right) \delta_{i j}+\operatorname{dev} \sigma_{i j}
$$

to be introduced into the Clausius-Duhem inequality (4), which now reads

$$
\sigma_{i j}^{\prime} \dot{\varepsilon}_{i j}-\phi\left(p_{a}-p_{w}\right) \frac{d S_{r}}{d t}-\left(\frac{1}{\rho_{a}} p_{a, k}-g_{k}\right) \mathrm{M}_{k}^{a}-\left(\frac{1}{\rho_{w}} p_{w, k}-g_{k}\right) \mathrm{M}_{k}^{w}-\frac{d \Psi_{s}}{d t} \geq 0 .
$$

The second term in the dissipation accounts for partial saturation by means of the suction (or capillary pressure), $s:=\left(p_{a}-p_{w}\right)$, which together with Bishop's stress describes the complete stress state. Analogously to the case of saturated porous media, the overall dissipation can be split into different contributions, one related to the skeleton, one to the wetting fluid (the linear involving $\mathrm{M}_{k}^{w}$ ) and the last one to the non-wetting gaseous mixture (the linear term involving $\mathrm{M}_{k}^{a}$ ). In the following we shall discuss the contribution relative to the solid skeleton, with the aim of capturing the main characteristics of the equations which govern the behavior of the unsaturated porous medium.

The dissipation of the gaseous phase is verified as an equality assuming the pressure of the gas to equal at any time $t$ the atmospheric reference pressure $p_{a}=0$. Moreover 
the fluid dissipation can be satisfied assuming Darcy's law to hold true, so that the corresponding governing equation for the saturation degree is provided by classical Richards' equation, which in the case of isotropic permeability, read as:

$$
\frac{d}{d t}\left(n S_{r}\right)=-\left[\frac{\varkappa k\left(S_{r}\right)}{\eta_{w}}\left(s_{, k}+\rho_{w} g_{k}\right)\right]_{, k} .
$$

Here $\varkappa$ is the intrinsic permeability of the skeleton and $\eta_{w}$ the dynamic viscosity of the wetting fluid. The so-called saturated permeability is defined by $k_{\text {sat }}:=\varkappa \rho_{w} g / \eta_{w}, g$ being the intensity of the gravitational acceleration. The isotropic permeability is prescribed by the product $k_{\text {sat }} k\left(S_{r}\right)$, where $k\left(S_{r}\right)$ is the relative permeability of the wetting fluid, see for more details [42].

\subsection{Dissipation within the solid skeleton}

The Clausius-Duhem inequality, reduced to account for the behavior of the partially saturated porous skeleton, i.e. of the solid grains and the liquid-gas interfaces, reads as follows:

$$
\Phi_{s}=\sigma_{i j}^{\prime} \dot{\varepsilon}_{i j}-\phi s \frac{d S_{r}}{d t}-\frac{d \Psi_{s}}{d t} \geq 0
$$

which implies the Helmholtz free energy $\Psi_{s}$ to depend on the elastic strain and the degree of saturation. For the sake of simplicity one can assume $\Psi_{s}$ to equal the summation of the elastic energy $\mathcal{E}$, stored into the solid skeleton during a reversible mechanical process, just depending on the elastic strain $\varepsilon_{i j}^{e}$, and the capillary energy $\phi U$, which is a function of the saturation degree, see [43]. Following the arguments proposed by [44] and more recently by [41], an additional contribution to the free energy can be provided by the so-called locked energy $\mathcal{Z}$, stored into the elastic skeleton when irreversible processes take place. Apparently this energy is parametrized by some hardening variable $\alpha$, defined in terms of the inelastic (plastic) strain. In the following we introduce this extra term in order to account for reversible variations of the free energy which can be achieved only when plastic hardening occurs; in particular we assume that this contribution is parametrized by the saturation degree. We therefore claim that:

$$
\Psi_{s}\left(\varepsilon_{i j}^{e}, S_{r} ; \alpha\right)=\mathcal{E}\left(\varepsilon_{i j}^{e}\right)+\phi U\left(S_{r}\right)+\mathcal{Z}\left(S_{r}, \alpha\right) .
$$

The elastic (reversible) part of the Bishop $\sigma_{i j}^{\prime e}$ stress tensor and the suction $s$ are given by

$$
\sigma_{i j}^{\prime e}=\frac{\partial \mathcal{E}}{\partial \varepsilon_{i j}^{e}}-U \delta_{i j}, \quad s=\left(p_{a}-p_{w}\right)=-\left(\frac{1}{\phi} \frac{\partial \mathcal{Z}}{\partial S_{r}}+\frac{\partial U}{\partial S_{r}}\right) .
$$

The constitutive law (10) 1 prescribing Bishop's stress does not depend only on the elastic strain of the solid skeleton but also on the saturation degree of the porous network. In other words the Terzaghi effective stress is not that of Bishop but, according with [43], the one given by the total stress $\sigma_{i j}$ plus the equivalent pore pressure $\pi \delta_{i j}:=\left(s S_{r}+U\right) \delta_{i j}$. As summarized in [43] the difference between the equivalent pore pressure $\pi$ and the average pressure $p^{*}=s S_{r}$, as well as that one between effective and Bishop's stress, becomes smaller and smaller when approaching saturation conditions. Equation $(10)_{2}$ provides the so-called WRC, characteristic of the fluid at equilibrium, within the regime of reversible 
processes; no hysteresis between wetting and drying processes is accounted for in this formula. The contribution provided by the locked energy $\mathcal{Z}$ yields a parametrization of the WRC by the hardening variable which in the following will be considered a function of the plastic strain. Accordingly the dependence of the WRC on irreversible changes in the void ratio, observed in several laboratory tests on soils, see e.g. [28], [32], [45] and [2], can be accounted for.

Coming back to the non-vanishing terms in the dissipation, the contributions to the Clausius-Duhem inequality, due to the behavior of the solid skeleton, are related on one hand to the evolution of the plastic deformations and the hardening parameters, on the other one to the capillary hysteresis, if any, see [41] and [35]. Thus one gets for the solid dissipation:

$$
0 \leq \Phi_{s}=p^{\prime} \dot{\varepsilon}_{v}^{p}+\operatorname{dev} \sigma_{i j} \dot{\varepsilon}_{i j}^{p}-\phi\left[s+\left(\frac{1}{\phi} \frac{\partial \mathcal{Z}}{\partial S_{r}}+\frac{\partial U}{\partial S_{r}}\right)\right] \frac{d S_{r}}{d t}+\beta \dot{\alpha}, \quad \beta:=-\frac{\partial \mathcal{Z}}{\partial \alpha}
$$

where $p^{\prime}$ is proportional to the first invariant of Bishop's stress tensor, $\sigma_{i j}^{\prime}$, and $\varepsilon_{v}^{p}$ is the volumetric plastic strain. Moreover $\beta$ is the force associated to the hardening variable $\alpha$, whilst the coefficient of the time derivative of the saturation degree accounts for hysteresis between the drainage and the imbibition curve in a suction cycle, if any.

In the following we focus on the case when the derivative of the locked energy with respect to the saturation degree can be neglected in comparison with the corresponding derivative of the capillary energy, which excludes any parametrization of the retention curve in terms of plastic deformations. This assumption will be discussed in more details in the following subsection where the Modified Cam-Clay model is extended to the case of partial saturation. On the other hand the dependence of $\beta$, conjugate to the hardening variable $\alpha$, on the saturation degree $S_{r}$ is still kept in the statement of the constitutive model. No wetting-drying cycle is simulated and consequently no contribution related to hydraulic hysteresis is provided to the dissipation of the skeleton; only wetting phenomena are taken into account, so that the capillary energy can be deduced from the wetting path of the WRC. Moreover, for the sake of simplicity, the equivalent pore pressure $\pi$ is assumed to coincide with the average pressure $p^{*}$; the more close to saturation the porous system is, the smaller the error will be.

The plastic model is formulated by considering, for the sake of simplicity, an isotropic loading function parametrized by the hardening force $\beta$ : $f\left(p^{\prime} ; q ; \beta\right) \leq 0$, where $q$ is the so-called deviator stress defined by the second invariant of the deviatoric effective stress $\left(J_{2}\right)$ as $q:=\sqrt{3 J_{2}}=3 \sqrt{\left(I_{1}^{2} / 3-I_{2}\right)}$. The principle of maximum plastic work holds true, which implies convexity of the yield surface $f=0$, for every fixed value of the hardening force. Normality of the plastic strain to the yield surface is expressed by the following flow rule:

$$
\dot{\varepsilon}_{v}^{p}=\Lambda \frac{\partial f}{\partial p^{\prime}}, \quad \operatorname{dev}\left(\dot{\varepsilon}_{i j}^{p}\right)=\Lambda \frac{\partial f}{\partial q} \frac{\partial q}{\partial\left(\operatorname{dev} \sigma_{i j}\right)},
$$

where the plastic multiplier $\Lambda$ obeys the Kuhn-Tucker conditions. The consistency condition, during plastic strains, allows for evaluating the expression of the plastic multiplier in terms of a proper hardening modulus $H$ and to characterize the hardening (softening) conditions. According with the adopted form of the locked energy $\mathcal{Z}$, consistency yields to:

$$
0=\dot{f}=\frac{\partial f}{\partial p^{\prime}} \dot{p}^{\prime}+\frac{\partial f}{\partial q} \frac{\partial q}{\partial\left(\operatorname{dev} \sigma_{i j}\right)} \operatorname{dev} \dot{\sigma}_{i j}-\frac{\partial f}{\partial \beta}\left(\frac{\partial^{2} \mathcal{Z}}{\partial \alpha^{2}} \dot{\alpha}+\frac{\partial^{2} \mathcal{Z}}{\partial \alpha \partial S_{r}} \dot{S}_{r}\right) .
$$


It is worth to notice that the flow rule for the hardening variable $\alpha$ is, in the general case, non-associated, which means that a suitable non-associated plastic potential $\varphi$ can be involved: $\dot{\alpha}=\Lambda \frac{\partial \varphi}{\partial \beta}$, see [27]. Consequently the following form of the plastic multiplier arises:

$$
\Lambda=\frac{1}{H}\left(\frac{\partial f}{\partial p^{\prime}} \dot{p}^{\prime}+\frac{\partial f}{\partial\left(\operatorname{dev} \sigma_{i j}\right)} \operatorname{dev} \dot{\sigma}_{i j}^{\prime}-\frac{\partial f}{\partial \beta} \frac{\partial^{2} Z}{\partial \alpha \partial S_{r}} \dot{S}_{r}\right), \quad H:=\frac{\partial f}{\partial \beta} \frac{\partial^{2} Z}{\partial \alpha^{2}} \frac{\partial \varphi}{\partial \beta} .
$$

The last term in the previous equation implies that increments of plastic strain are constitutively related not only to variations of stress but also to changes in saturation degree. Since $\Lambda$ must be non-negative, positive plastic strains can arise, for positive values of $H$, when the stress increment is oriented outwards with respect to the current elasticity domain, keeping constant $S_{r}$, or when positive variations of the degree of saturation are accompanied by a positive change of the hardening force $\beta$ with respect to saturation, keeping constant the state of stress, and taking the derivative of $f$ with respect to $\beta$ to be positive. Apparently the opposite is true for softening, which occurs when $H<0$.

\subsection{Extending the Modified Cam-Clay model}

Following [26] and [27, 46], we adopt the Modified Cam-Clay model extended to the case of partially saturated soils. A brief resumé of this formulation is reported in the following; we refer to the above cited papers for more details. In particular we assume the yield locus prescibed in terms of Bishop's stress components by the following ellipse in the $\left(p^{\prime}, q\right)$ plane:

$$
f_{c c}=q^{2}+M^{2} p^{\prime}\left(p^{\prime}-p_{c}^{\prime}\right)=0,
$$

and consider a parametrization of the consolidation effective pressure $p_{c}^{\prime}$ by the degree of saturation; as usual $M$ indicates the slope of the Critical State Line (CSL) .

According with the above general framework, an associated flow rule is assumed for plastic strains, the evolution of which is therefore prescibed by the derivative with respect to stress of the associated potential $f_{c c}$ :

$$
\dot{\varepsilon}_{v}^{p}=\Lambda \frac{\partial f_{c c}}{\partial p^{\prime}}=\Lambda M^{2}\left(2 p^{\prime}-p_{c}^{\prime}\right), \quad \operatorname{dev} \dot{\varepsilon}_{i j}^{p}=\Lambda \frac{\partial f_{c c}}{\partial q} \frac{\partial q}{\partial\left(\operatorname{dev} \sigma_{i j}\right)}=3 \Lambda \operatorname{dev} \sigma_{i j} .
$$

Assuming the hardening internal variable to coincide with the volumetric plastic strain, $\alpha=\varepsilon_{v}^{p}$, and the corresponding force to equal the opposite of the consolidation effective stress, $\beta=-p_{c}^{\prime}$, a state equation, linking $p_{c}$ with $\varepsilon_{v}^{p}$, need now to be formulated. Following [27], and extending the Modified Cam-Clay saturated model, see e.g. [38], the time evolution of $p_{c}^{\prime}$ is defined in terms of a double-hardening mechanism:

$$
\dot{p}_{c}^{\prime}=\dot{p}_{c(\text { sat })}^{\prime}+\dot{p}_{c(\text { unsat })}^{\prime}
$$

with

$$
\dot{p}_{c(\text { sat })}^{\prime}=\frac{v p_{c}^{\prime}}{\lambda-\kappa} \dot{\varepsilon}_{v}^{p}, \quad \dot{p}_{c(\text { unsat })}^{\prime}=-b p_{c}^{\prime} \dot{S}_{r}
$$

Here $v=1+e=1 /(1-n)$ is the specific volume, given in terms of the void ratio $e$ and the porosity $n ; \kappa$ and $\lambda$ are the slopes of the unloading-reloading line and of the Normal 
Consolidation Line (NCL), both in a semi-logarithmic picture in the compressibility plane. Equations (17) and (18) imply:

$$
-\beta=p_{c}^{\prime}=p_{c}^{\prime 0} \exp \left(\frac{v}{\lambda-\kappa} \Delta \varepsilon_{v}^{p}\right) \exp \left(-b \Delta S_{r}\right),
$$

$\Delta \varepsilon_{v}^{p}$ and $\Delta S_{r}$ being the increments of the plastic strain and the degree of saturation with respect to suitable reference values. According with equation (11) a prescription for the locked energy $\mathcal{Z}$, consistent with (19), can be formulated as

$$
\mathcal{Z}\left(S_{r}, \varepsilon_{v}^{p}\right)=\left(\frac{\lambda-\kappa}{v}\right) p_{c}^{\prime}+f\left(S_{r}\right)
$$

where $f$ is a function only of the saturation degree. Consider now the constitutive characterization of the WRC, given by equation $(10)_{2}$; the contribution of the derivative of the locked energy with respect to $S_{r}$ is

$$
\frac{1}{\phi} \frac{\partial \mathcal{Z}}{\partial S_{r}}=-\frac{b}{\phi}\left(\frac{\lambda-\kappa}{v}\right) p_{c}^{\prime}+\frac{1}{\phi} \frac{d f}{d S_{r}}
$$

which should account for the effect of irreversible changes in the void ratio on the retention characteristics of the soil. Assuming this effect negligible, in the absence of plastic strain, constraints the derivative of $\mathcal{Z}$ to vanish when $\Delta \varepsilon_{v}^{p}=0$, so that

$$
\frac{d f}{d S_{r}}=p_{c}^{\prime 0} b\left(\frac{\lambda-\kappa}{v}\right) \exp \left(-b \Delta S_{r}\right) \quad \Rightarrow \quad f\left(S_{r}\right)=-p_{c}^{\prime 0} b^{2}\left(\frac{\lambda-\kappa}{v}\right) \exp \left(-b \Delta S_{r}\right) .
$$

Coming back to the general case, the contribution to the WRC provided by the locked energy is therefore given by

$$
\frac{1}{\phi} \frac{\partial \mathcal{Z}}{\partial S_{r}}=-\frac{b}{\phi}\left(\frac{\lambda-\kappa}{v}\right)\left[p_{c}^{\prime}-p_{c}^{\prime 0} \exp \left(-b \Delta S_{r}\right)\right]
$$

which filters out the effect of the variations of the saturation degree but is only affected by the variations of the volumetric plastic strain. This justifies, within the framework of small deformations, the hypothesis of neglecting this contribution with respect to the derivative of the capillary energy $U$ in equation $(10)_{2}$.

Equation (14) for the plastic multiplier reads now as follows:

$$
\Lambda=\frac{1}{H}\left[2 M^{2}\left(p^{\prime}-\frac{1}{2} p_{c}^{\prime}\right) \dot{p}^{\prime}+\operatorname{dev} \sigma_{i j} \operatorname{dev} \dot{\sigma}_{i j}^{\prime}-M^{2} p^{\prime} \frac{\partial p_{c}^{\prime}}{\partial S_{r}} \dot{S}_{r}\right], \quad H=2 M^{4} p^{\prime}\left(p^{\prime}-\frac{1}{2} p_{c}^{\prime}\right) \frac{\partial p_{c}^{\prime}}{\partial \epsilon^{p}} .
$$

The material exhibits therefore a hardening behavior when $p^{\prime}>p_{c}^{\prime} / 2$, which corresponds to $H>0$, and a softening behavior when $p^{\prime}<p_{c}^{\prime} / 2$, which corresponds to $H<0$. As already noticed, in the general formulation, hardening can occur when the stress increment is oriented outwards with respect to the current elasticity domain, keeping constant $S_{r}$, or in case of wetting, when the stress is kept constant. In these last circumstances the only non-vanishing contribution to $\Lambda$ comes from the last term in equation $(24)_{1}$, which is indeed positive when $\dot{S}_{r}>0$ (wetting), taking into account the negative sign of the derivative of the consolidation stress with respect to the degree of saturation, see (19). This condition models the phenomenon of collapse by wetting typical of unsaturated soils. 
Substituting the flow rule (16) into the dissipation inequality (11), taking into account the assumptions for the hardening variables and bearing in mind the expression for the yield surface provide the following reduced expression for the Clausius-Duhem inequality:

$$
\Phi_{s}=\Lambda M^{2}\left(p_{c}^{\prime}-p^{\prime}\right) p^{\prime}
$$

which is indeed consistent with thermodynamics and similar to that of the modified CamClay model, see [47].

\section{Calibration of the Modified Cam-Clay model}

The Modified Cam-Clay constitutive model has been calibrated by means of laboratory tests performed under suction controlled conditions on a volcanic ash and a clayey silt, say the Cervinara ash and the Jossigny silt, according with the sites (south on Naples, Italy, and east of Paris, France), where they have been taken. The two materials significantly differ in the physical characteristics as well as in the main hydro-mechanical properties, in particular the initial void ratio, the WRC and the compressibility.

\subsection{Cervinara ash}

The volcanic ash from Cervinara is a silty sand with a non-plastic fine-grained component, generally less than $30 \%$, constituted by very poor ordered minerals (sanidine and pyroxenes) see $[48,36,5]$. This material is characterized by macropores having a size comparable to that of the coarsest particles and it has an uniformity coefficient, $d_{60} / d_{10}$, equal to 10 and an in-situ porosity ranging between 0.65 and 0.75 .

The parameters of the Van Genuchten type WRC, $S_{r}=\bar{S}_{r}+\left(1-\bar{S}_{r}\right) /\left(1+(\alpha s)^{n}\right)^{m}$, where $\bar{S}_{r}$ is the residual saturation degree, have been calibrated with respect to the experimental results obtained under suction controlled conditions and reported in [36]. A comparison between the Van Genuchten curve and the experimental data is reported in Figure 1a. Consider now the permeability which depends in partially saturated porous media not only on the pore-structure, say the void ratio, and the pore-fluid properties, say the density and the viscosity, but also on the relative amount of pore-fluid in the system, say the degree of saturation. Here the unsaturated hydraulic permeability is fitted with

the Van Genuchten model $k\left(S_{r}\right)=\sqrt{S_{r}}\left[1-\left(1-S_{r}^{\delta}\right)^{\beta}\right]^{n}$, see Figure 1b. The parameters $\delta, \beta$ and $n$ have been calibrated by back analysing the equalisation stage of triaxial tests related to different values of suction, considering in particular the time evolution of the water content, see Figure 2a. The mechanical properties of the material have been calibrated following the procedure reported in [2], for oedometric conditions, and extended to triaxial conditions in [49]. The slopes of the NCL and of the unloading-reloading line in the plane $v-l n p^{\prime}$, say $\lambda$ and $\kappa$ respectively, are calibrated with the results of the isotropic compression tests in saturated conditions, see Figure $2 \mathrm{~b}$. The parameter $b$, which describes translations of the NCL due to partial saturation, is calibrated using the results of the isotropic compression test, in particular at $s=40 \mathrm{kPa}$, see [36]. The comparison between the experimental results and the model predictions is reported in Figure $2 \mathrm{~b}$. It confirms the capability of the model to describe the mechanical behavior of the soil along the stress paths applied during compression tests. Parameter $M$ has been calibrated on 


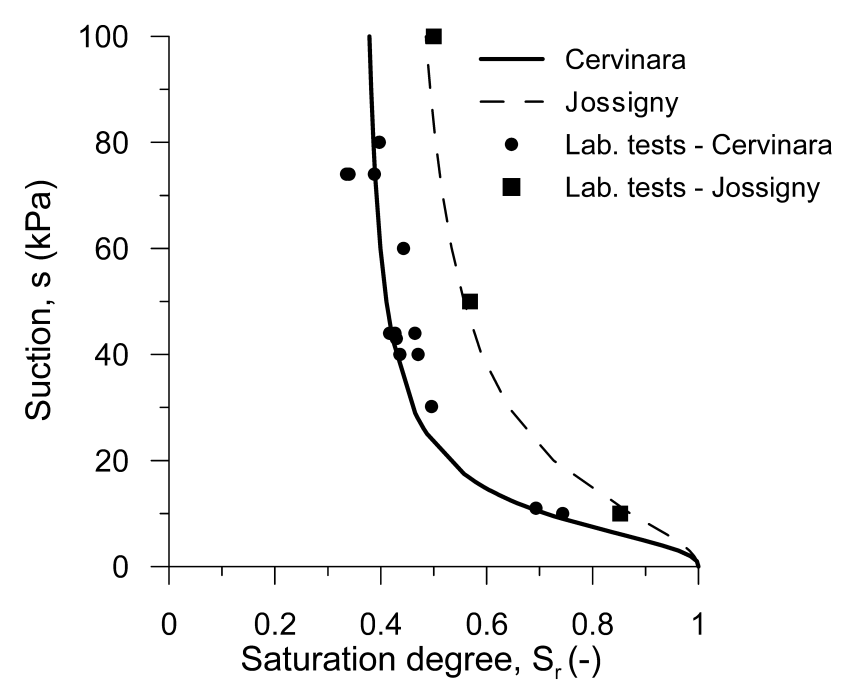

(a)

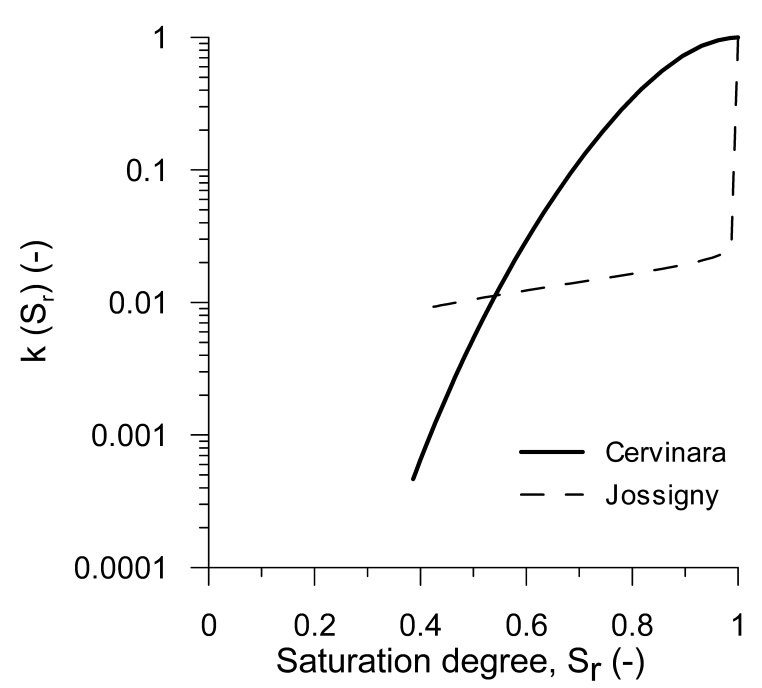

(b)

Figure 1: (a) WRCs fitted by the Van Genuchten model; (b) Permeabilities back analyzed by the Van Genuchten model.

the basis of the shear strength obtained in triaxial tests at different confining pressures and a typical value for the Poisson's ratio has been assumed.

\subsection{Jossigny silt}

The Jossigny silt has been studied in saturated and unsaturated conditions by several authors, see e.g. $[32,50]$. It is a low plasticity silt with $25 \%$ of clay retrieved by an alluvial deposit located at Jossigny, East of Paris, France. Clay minerals determined by X-ray diffractometry are illite, kaolinite and inter-stratified illite-smectite. No significant swelling properties on compacted states have been observed on wetting [37].

The parameters of the model have been calibrated at the light of the experimental results under saturated and unsaturated conditions obtained in [50] and reported in [51]. The parameters of the Van Genuchten type WRC have been calibrated by best fitting the wetting path performed under suction controlled conditions at an average void ratio $e=0.82$, see Figure 1a. The relative permeability curve is taken from the one provided by [52], see Figure $1 b$.

The compressibility parameters $\lambda, \kappa$ and $b$ have been calibrated on the basis of the experimental results obtained during oedometer loading under controlled suction. The comparison between the model prediction and the data is reported in Figure 3. The axial loading stage of the triaxial tests under unsaturated condition has been used to calibrate the slope of the CSL in the $\left(p^{\prime}, q\right)$-plane, say $M$.

The hydro-mechanical parameters of both materials are summarized in Table 1 and Table 2.

\section{Numerical modeling of water imbibition}

The objective of the numerical modeling is to discuss the imbibition of a two-dimensional soil column with geostatic stresses distribution with null lateral deformation. This prob- 


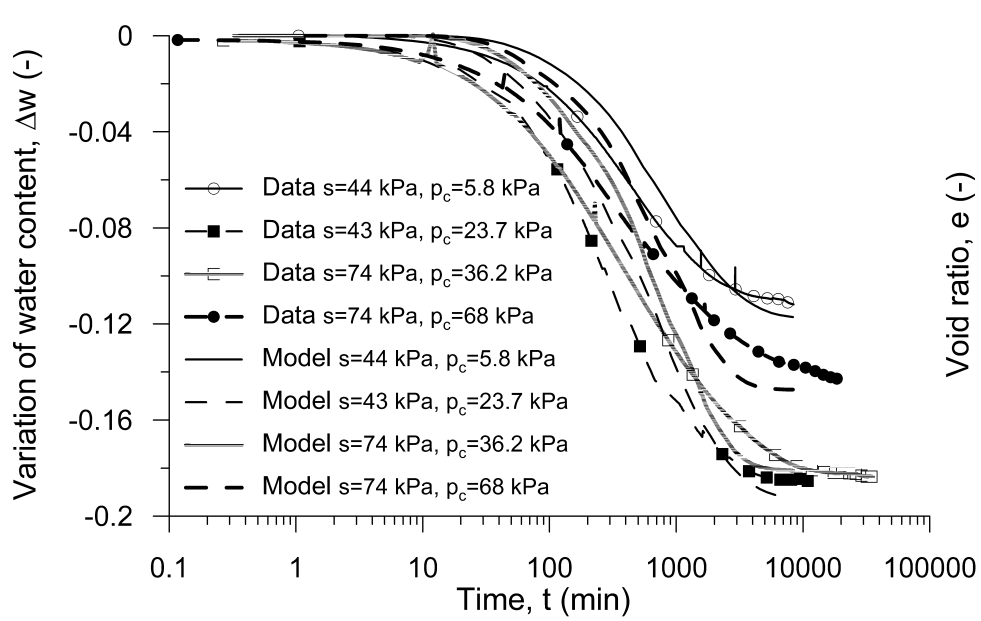

(a)

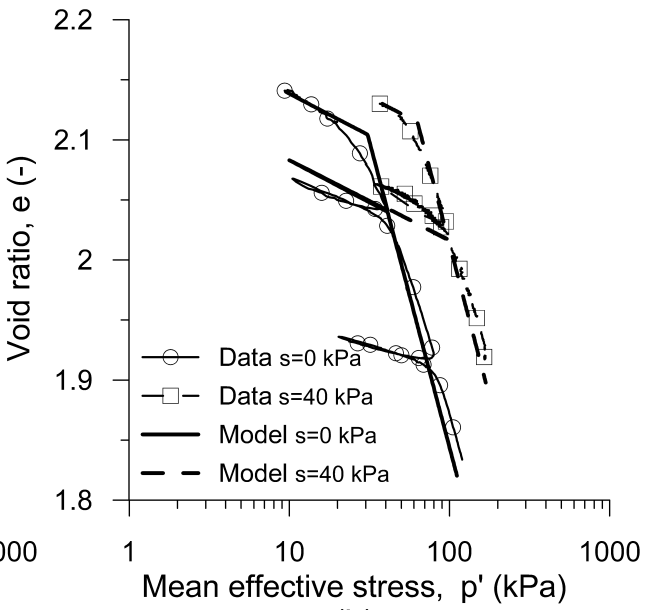

(b)

Figure 2: Fitting of the hydro-mechanical constitutive parameters for the Cervinara ash: (a) water content evolution during the equalisation stage of different triaxial tests; (b) void ratios in terms of mean effective stress during isotropic compression tests

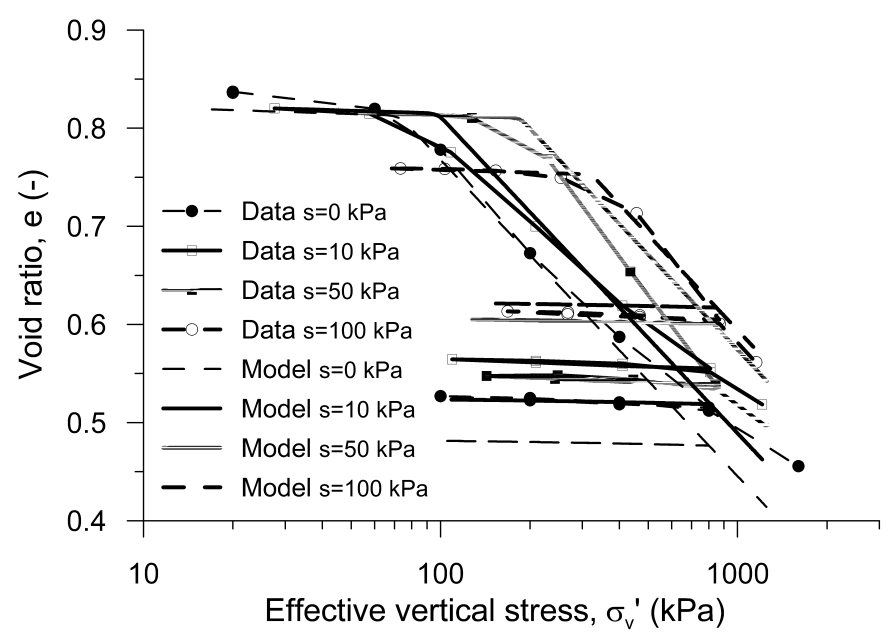

Figure 3: Fitting of the mechanical parameters: void ratio in terms of vertical effective stress during oedometric compression tests of the Jossigny silt.

lem simulates the effect of rainfall events with uniform spatial distribution over a large ground surface.

The soil imbibition is analyzed by performing plane strain numerical simulations with the commercial code ABAQUS/Standard. The Modified Cam-Clay model (15)-(24) has been implemented in this code by [53], adopting the implicit return mapping algorithm developed by [54]. The subroutine has been initially validated simulating the response of a reference elementary volume, see [53], and later in the study of a real dam, see [55].

The adopted two-dimensional finite element mesh is $H=10 \mathrm{~m}$ high and $B=2 \mathrm{~m}$ wide, see Figure 4. The numerical analysis returns stable and accurate responses which do not appreciably depend on element size. Mechanical boundary conditions are of three types: prescribed null horizontal displacements on the lateral sides of the mesh; prescribed null total displacements at the bottom of the column and prescribed null stress on the top of the column. Hydraulic conditions impose null water flux at all boundaries except on 


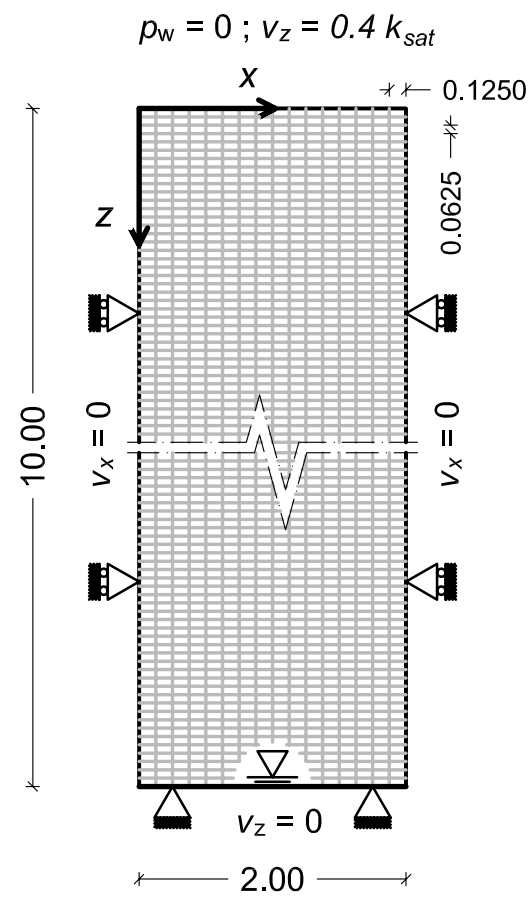

Dimension in $\mathrm{m}$

Figure 4: The finite element mesh consists of fine 8-noded quadrilateral elements.

the top of the mesh where two types of infiltration are allowed, see Figure 4 . The first one simulates the case of an intense rainfall for which ground surface becomes saturated. This case is modeled by applying null water pressure on the top of the mesh and corresponds to fix the so-called surface ponding condition. The second one corresponds to the case of a moderate rainfall intensity, equal to $40 \%$ of the saturated permeability of the soil, see Table 3.

Under geostatic conditions, the vertical total stress $\sigma_{v}$ increases almost linearly with depth. The pore-water pressure profile is assumed hydrostatic and the groundwater table is initially located at the lower boundary. The effective horizontal stresses $\sigma_{h}^{\prime}$ are expressed as a function of $\sigma_{v}^{\prime}$ through the earth pressure coefficient $K_{0}$, see [56].

\section{Numerical results}

\subsection{Hydration and deformation profiles}

The main features of the response of the two materials can be characterized following the development of the imbibition process of the layer. To this aim, the time evolutions of $S_{r}, p_{w}$ and $\varepsilon_{v}^{p}$ profiles are depicted in Figures 5-8 for the pyroclastic and clayey soils, respectively, for the two infiltration conditions reported in Table 3 . In the case of surface ponding condition the simulation is carried out until reaching the stationary conditions whereas it is sharply interrupted when water table reaches the ground surface in case of the flow boundary condition. 


\subsubsection{Cervinara ash}

$S_{r}, p_{w}$ and $\varepsilon_{v}^{p}$ profiles are depicted in Figure 5, for surface ponding boundary conditions, at different dimensionless times $T=t k_{\mathrm{sat}} / H$, whose definition naturally arises from the dimensional analysis of Richards' equation (7) specialized by the one-dimensional problem under consideration. As presented by [57], infiltration takes place along a sharp front; this is due to a specific range for the ratio between the unsaturated permeability and the slope of the retention curve, which prevails, see Figure 5a. When the wetting front reaches the bottom of the layer, the pore-water pressure becomes positive, the water table moves upwards through the layer and the hydrostatic conditions are quickly attained, see Figure $5 \mathrm{~b}$. On the other hand no positive values of the pore-water pressure are achieved during the downward propagation of the wetting front. Plastic volumetric strains also show up when the front deepens, with a maximum at the current front locus, while decreasing to a mostly constant value below it. Once attained complete saturation along the profile, no significant increase of plastic volumetric strain arises.

As far as concerns flow controlled infiltration, $S_{r}$ profiles indicate slower and more diffuse front propagation and full saturation is reached first at the bottom of the layer, see Figure 6a. From that time, water level starts to rise up to the top of the column leading to a decrease of vertical effective stress at almost constant vertical total stress, see Figure 12. During downward front propagation ( $T$ ranging from 0.04 to 0.24 ), the volumetric plastic strain profile moves with front advance and exhibits a maximum value above the front. During the further rise of water table, additional volumetric plastic strain develops and the plastic volumetric strain profile is characterized by a parabolic shape with maximum value at $60 \%$ of column depth.

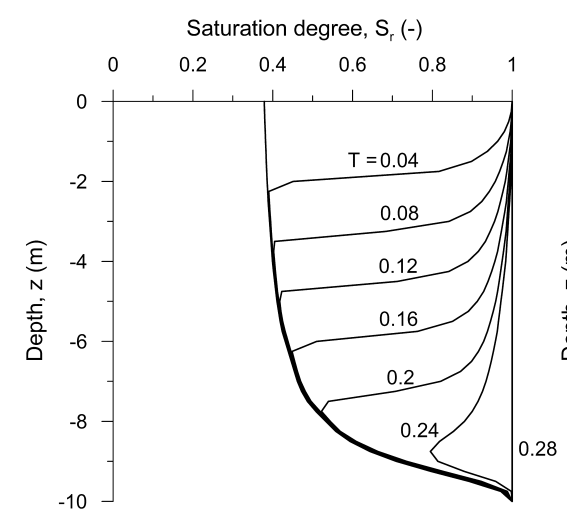

(a)

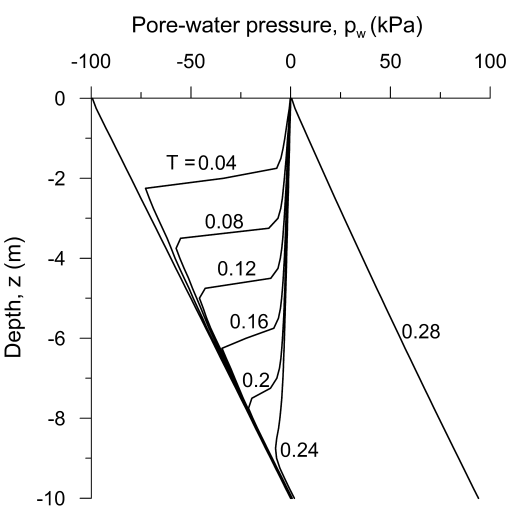

(b)

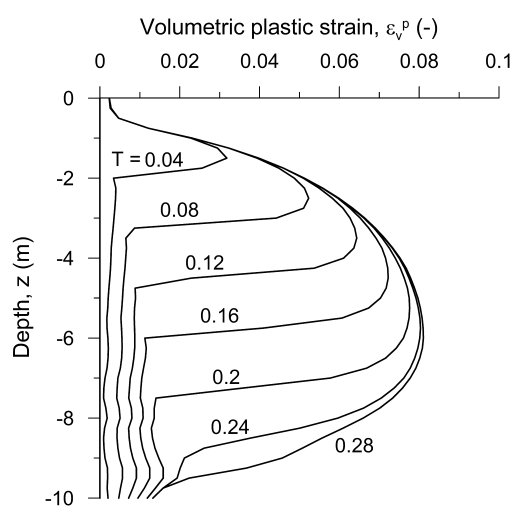

(c)

Figure 5: Water saturation profiles relative to Cervinara ash: isochrones of $S_{r}, p_{w}$ and $\varepsilon_{v}^{p}$, for surface ponding boundary conditions, parametrized by the dimensionless time $T$.

\subsubsection{Jossigny silt}

The profiles of $S_{r}, p_{w}$ and $\varepsilon_{v}^{p}$, for the case of Jossigny silt, for surface ponding boundary conditions, are reported in Figure 7. Besides the qualitative similarity with those of Cervinara's ash, there are quantitative differences in the saturation degree and the porewater pressure (wetting front is thicker and infiltration quicker in Jossigny silt) because of the absolute values of permeability and WRC. As in the case of Cervinara ash, the profiles 


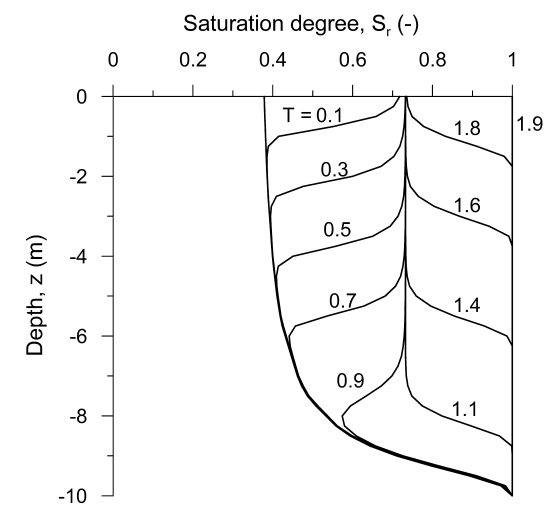

(a)

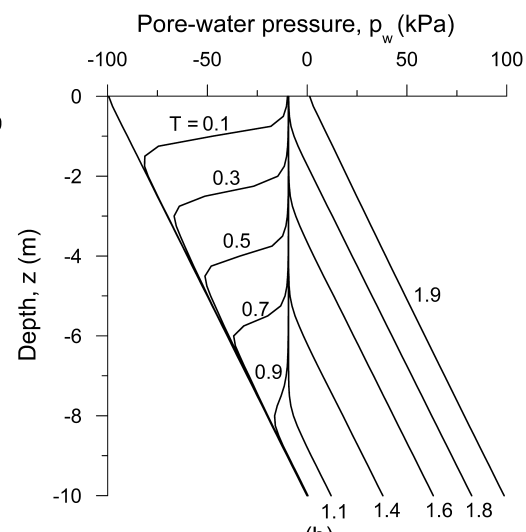

(b)

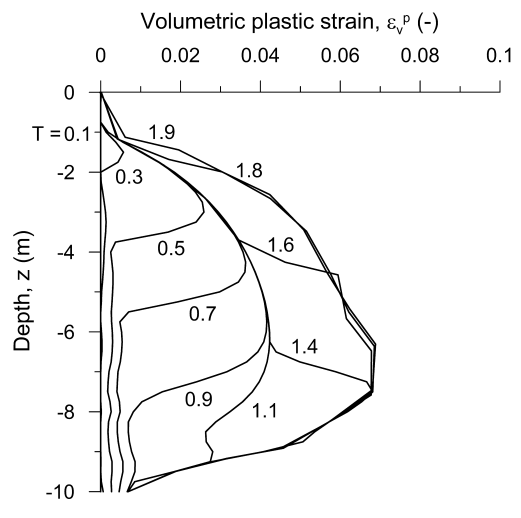

(c)

Figure 6: Water saturation profiles relative to Cervinara ash: isochrones of $S_{r}, p_{w}$ and $\varepsilon_{v}^{p}$, for flow boundary conditions, parametrized by the dimensionless time $T$.

of $\varepsilon_{v}^{p}$ have a maximum at the current placement of the wetting front and a progressively decrease above and below it.

As far as concerns flow boundary conditions, the case of the silt is quite similar to that of the ash. However because of the differences in the $k\left(S_{r}\right)$ curves the degree of saturation is quite close to one, at the top of the layer. Therefore the second part of the evolution, say the rising up of the saturation front from the bottom, is almost undedectable.

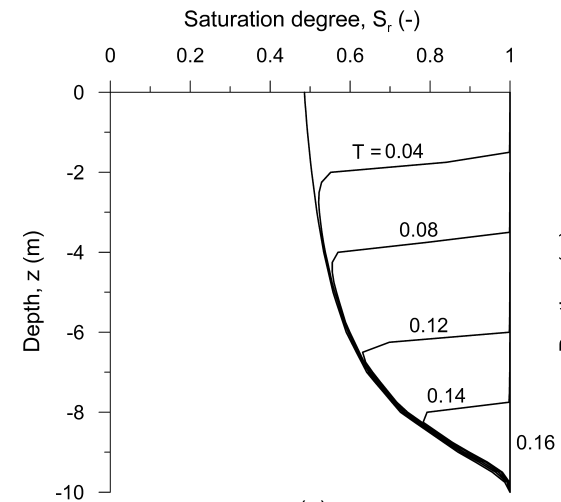

(a)

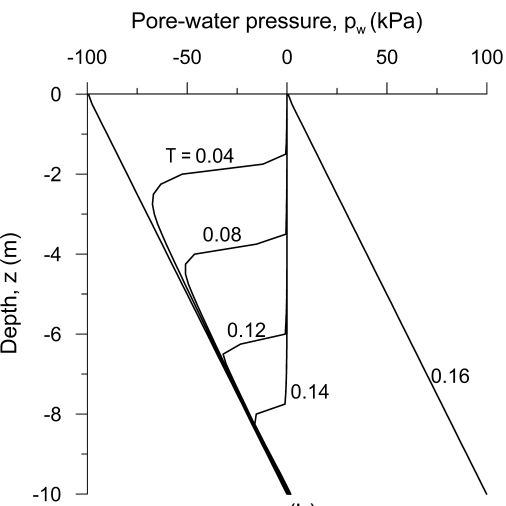

(b)

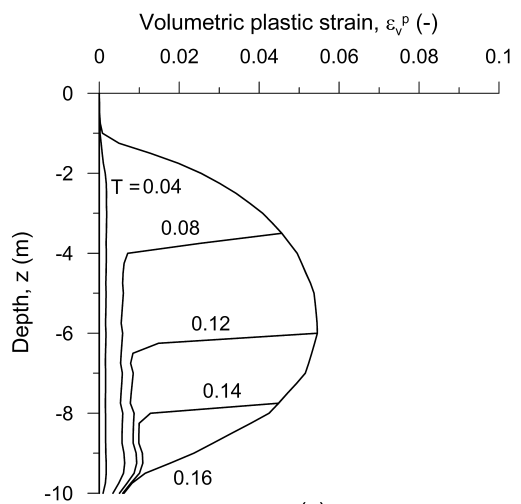

(c)

Figure 7: Water saturation profiles relative to Jossigny silt: isochrones of $S_{r}, p_{w}$ and $\varepsilon_{v}^{p}$, for surface ponding boundary conditions, parametrized by the dimensionless time $T$.

\subsubsection{Settlements induced by wetting}

In Figure 9, the settlements induced by rainfall infiltration at the surface are reported as function of the dimensionless time. As expected, the evolution of settlements due to plastic compression is faster when prescribing ponding rather than flow boundary conditions, for both materials. The maximum of displacement $w=w_{\max } \simeq 0.33-0.44 \mathrm{~m}$ is attained, at ponding, for $T=0.16$ and 0.28 for Jossigny and Cervinara soil, respectively.

Under flow boundary conditions, the magnitude of the maximum displacement in the Jossigny silt is the same as that achieved at ponding, but with a time delay, $T \simeq 0.8$. In the Cervinara ash the double mechanism of wetting during flow controlled infiltration induces plastic deformation smaller than that relative to surface ponding: $w_{\max } \simeq 0.38$ 


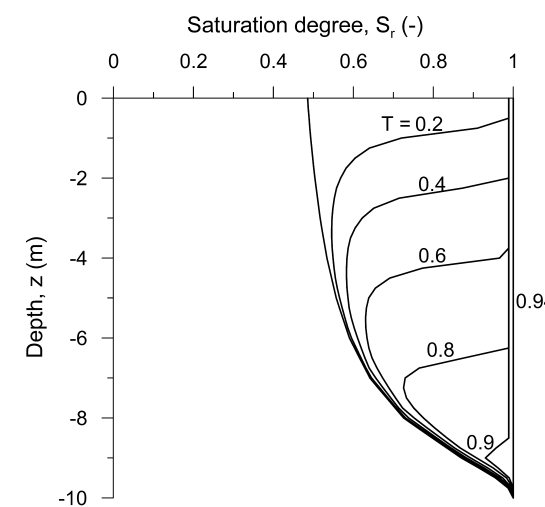

(a)

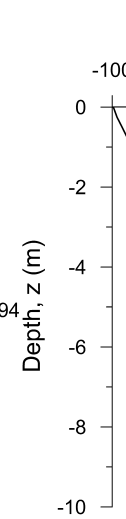

Pore-water pressure, $\mathrm{p}_{\mathrm{w}}(\mathrm{kPa})$

$\begin{array}{llll}-50 & 0 & 50 & 100\end{array}$

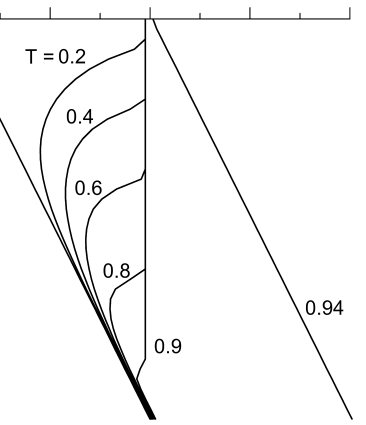

(b)

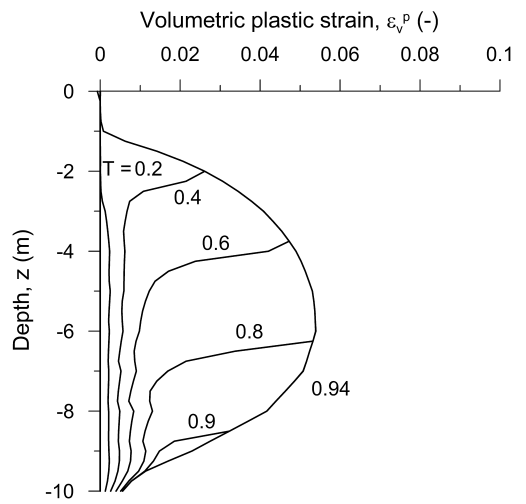

(c)

Figure 8: Water saturation profiles relative to Jossigny silt: isochrones of $S_{r}, p_{w}$ and $\varepsilon_{v}^{p}$, for flow boundary conditions, parametrized by the dimensionless time $T$.

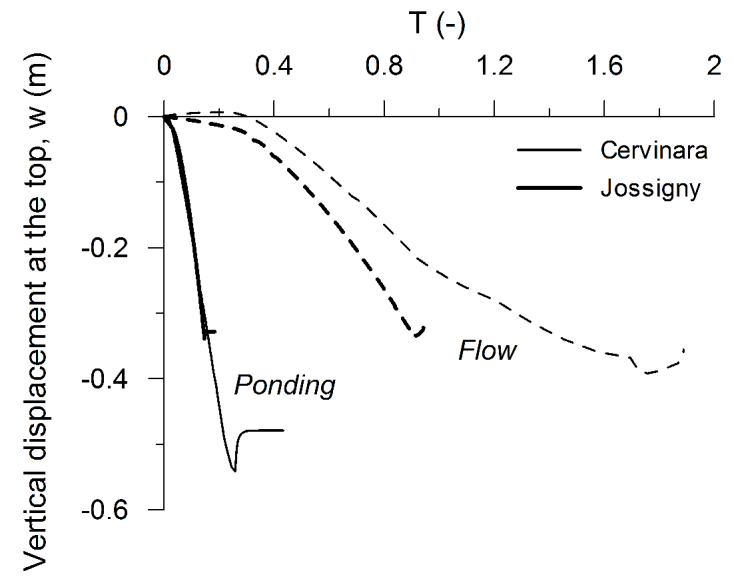

Figure 9: Settlements induced by imbibition for the Cervinara ash and the Jossigny silt.

$\mathrm{m}(T \simeq 1.8)$. The late swelling observed in the four cases is related to the elastic part of the deformation induced by the decrease of effective stress.

\subsection{Coupled hydro-mechanical responses at different depths}

The hydro-mechanical behaviors of the Cervinara and Jossigny soils are analysed in the following subsections, focusing the attention on the responses of two reference elements located at depths of 2 and $8 \mathrm{~m}$ from the top of the soil domain. The time evolutions of the saturation degree, the pore-water pressure, the unit soil weight, the plastic volumetric strains, the increments of the total and effective vertical and horizontal stresses and the stress paths are reported in order to monitor the behavior of the soil close to the surface and the bottom of the layer, when surface ponding and flow boundary conditions are imposed at the top of the domain. The increments in the vertical and horizontal stresses are evaluated with respect to the geostatic values.

\subsubsection{Cervinara ash}

The time evolution of the saturation degree $S_{r}$ at the above mentioned elements when null water pressure is imposed at the top of the layer is shown in Figure 10. For surface 

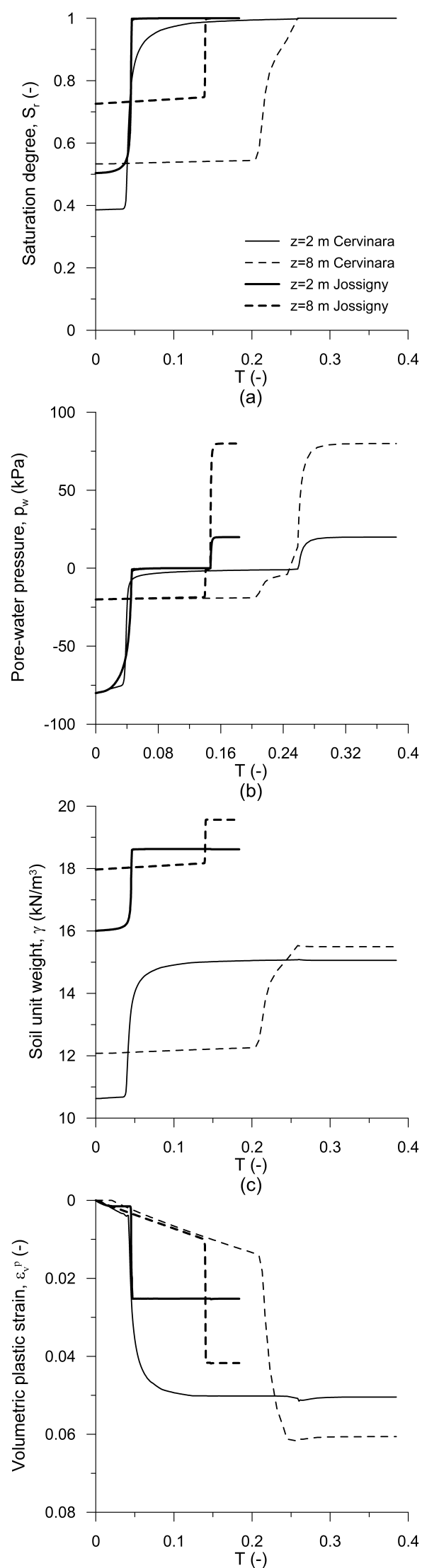

(d)

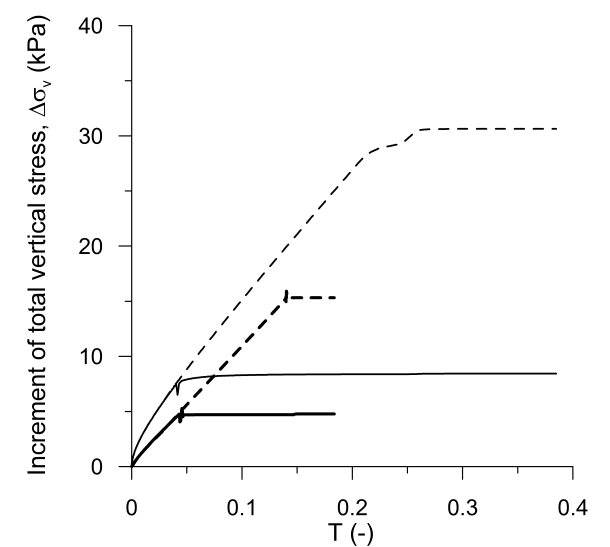

(e)
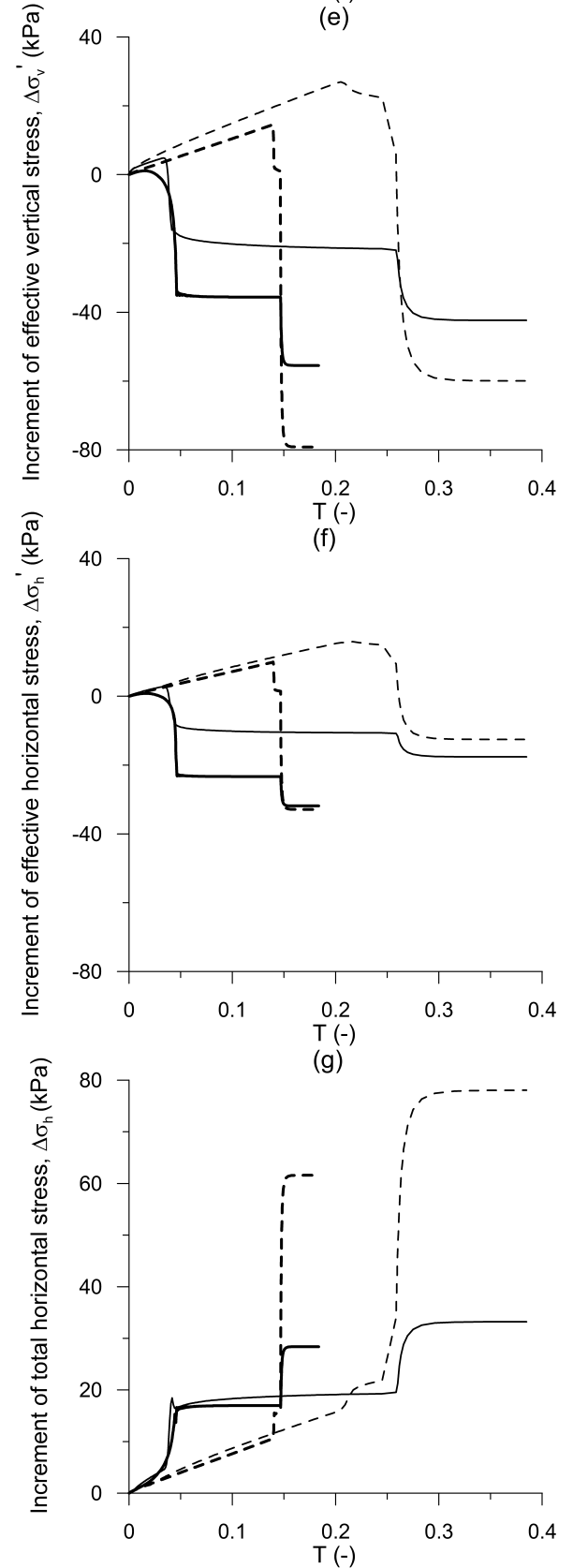

(h)

Figure 10: Time evolution of $S_{r}, p_{w}, \gamma, \varepsilon_{v}^{p}, \Delta \sigma_{v}, \Delta \sigma_{v}^{\prime}, \Delta \sigma_{h}^{\prime}, \Delta \sigma_{h}$ for surface ponding boundary conditions. 

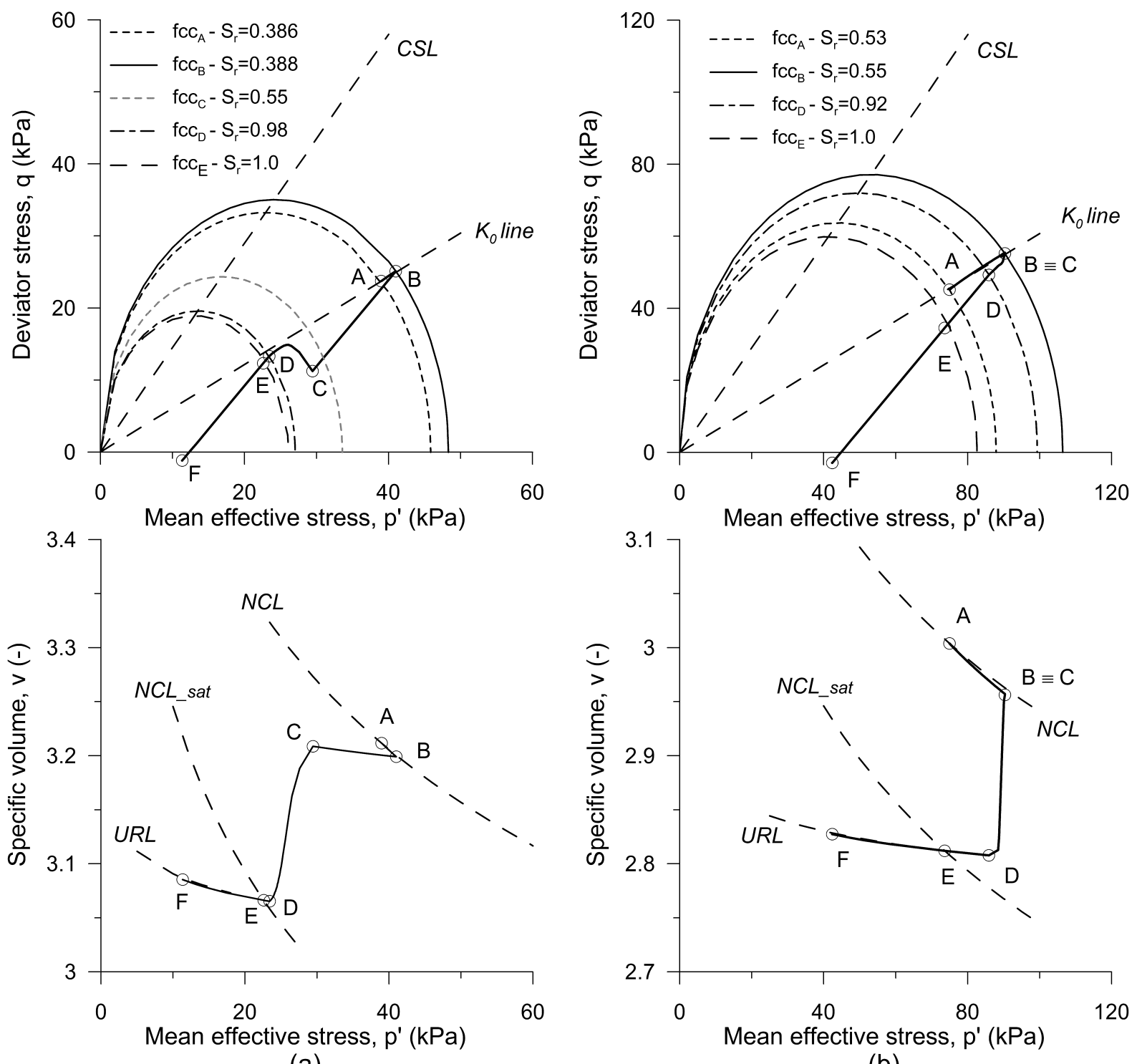

(a)

(b)

Figure 11: Stress paths and volumetric behavior for surface ponding boundary conditions for Cervinara ash: (a) shallow element: $z=2 \mathrm{~m}$; (b) deep element: $z=8 \mathrm{~m}$. 
ponding conditions, $S_{r}$ keeps constant values until the wetting front reaches the reference element, which is in agreement with the saturation profiles presented in Figure 5a. As the wetting front passes through it, a smooth variation of $S_{r}$ is observed and saturation is attained rather slowly.

The pore-water pressure evolves in time according with the profiles reported in Figure 10b. Afterwards, $p_{w}$ increases abruptly when the wetting front reaches the reference element. It remains then almost equal to zero up to the time for which the wetting front reaches the bottom of the layer. After this moment, the water table moves up from the bottom to the ground surface. When it reaches the reference element, the pore pressure starts to rise up again attaining its hydrostatic stationary value.

The time evolution of the unit soil weight is reported in Figure 10c. It maintains constant values until the wetting front is still above the reference element. As the wetting front passes through the element, the unit soil weight attains its stationary value.

The changes in time of the plastic strain $\varepsilon_{v}^{p}$ are drawn in Figure 10d. Again, when the wetting front is still above the reference element, the solid skeleton compresses according to the increment of the gravity loading exerted on it. When the front reaches the element, volumetric collapse occurs, causing irreversible volumetric strains, even when the mean effective stress decreases. Finally, as soon as the front attains the bottom of the soil domain and the water table rises through the reference element, the volumetric plastic strain shows no significant variations. This means that the response of the material in the last part of the imbibition process is substantially elastic.

The variations in time of the total vertical stress increment $\Delta \sigma_{v}$ induced by the infiltration process in the shallow and deep elements are shown in the Figure $10 \mathrm{e}$. As $\Delta \sigma_{v}$ responds to the cumulative gravity loading exerted on the element, it increases until the saturation conditions are attained above the element itself. This occurs in the Cervinara soil when the water table rises through the reference element.

The changes in time of the effective vertical stress increment $\Delta \sigma_{v}^{\prime}$ are shown in Figure 10f. $\Delta \sigma_{v}^{\prime}$ increases almost linearly with time until the wetting front reaches the position of the reference element. During this part of the evolution process, the profile of $\Delta \sigma_{v}^{\prime}$ corresponds exactly to that of $\Delta \sigma_{v}$. When the front passes through the element, the effective vertical stress decreases and remains constant until the front reaches the bottom of the domain. When the water table, moving from the bottom, reaches the reference element, $\Delta \sigma_{v}^{\prime}$ starts to decrease again attaining the stationary value, following the variation of the pore-water pressure.

The time evolution of the effective horizontal stress increment $\Delta \sigma_{h}^{\prime}$ is qualitatively similar to that of $\Delta \sigma_{v}^{\prime}$, even if it ranges over a thinner interval, see Figure $10 \mathrm{~g}$.

Finally the time evolution of the total horizontal stress increment $\Delta \sigma_{h}$ is shown in Figure 10h. The initial increment of $\Delta \sigma_{h}$ is due to that of $\Delta \sigma_{h}^{\prime}$; the subsequent increments are essentially caused by the positive variations of the pore-water pressure.

On the basis of the time evolution of the mechanical and hydraulic variables, which has been discussed above, one can also follow the stress paths relative to the shallow and the deep elements, see Figure 11. The evolution of the stress state is drawn in the $\left(p^{\prime}, q\right)$-plane emphasizing the relative position of the current stress state with respect to the current yield surface. Moreover the evolution of the specific volume is depicted in the $\left(p^{\prime}, v\right)$-plane together with the NCLs relative to the initial and saturated conditions and the final Unloading -Reloading Line (URL). The initial stress state $A$ lies on the yield surface along the $K_{0}$-line, as expected in a normally consolidated soil. In oedometric 

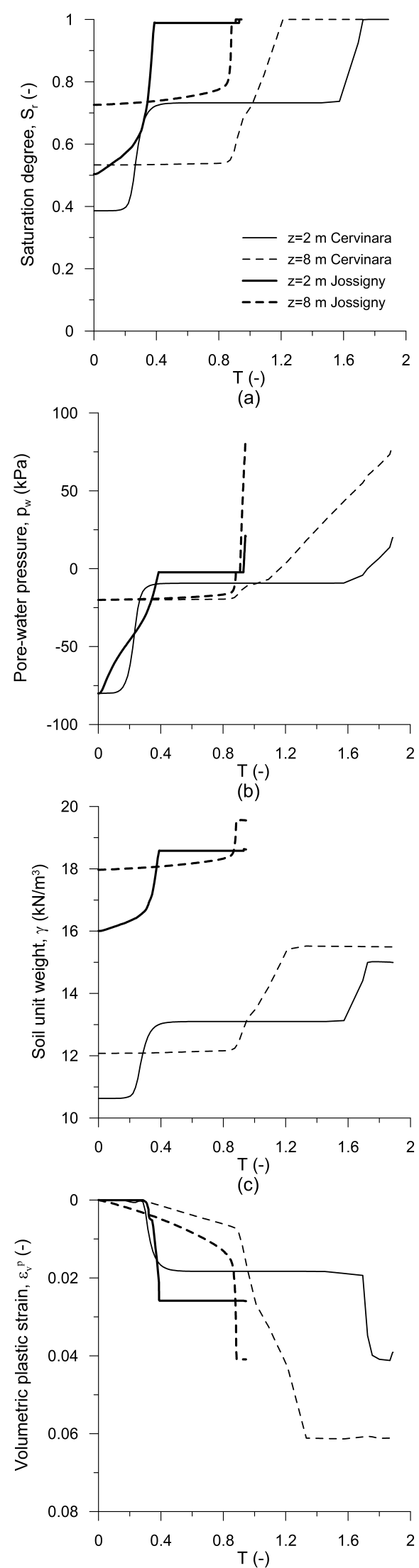

(d)

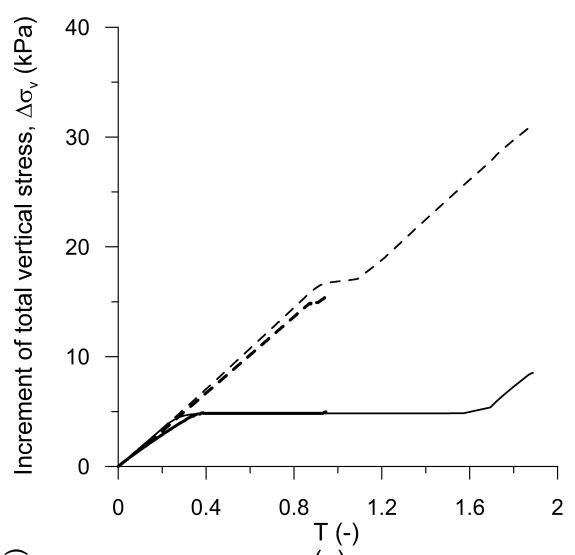

(e)

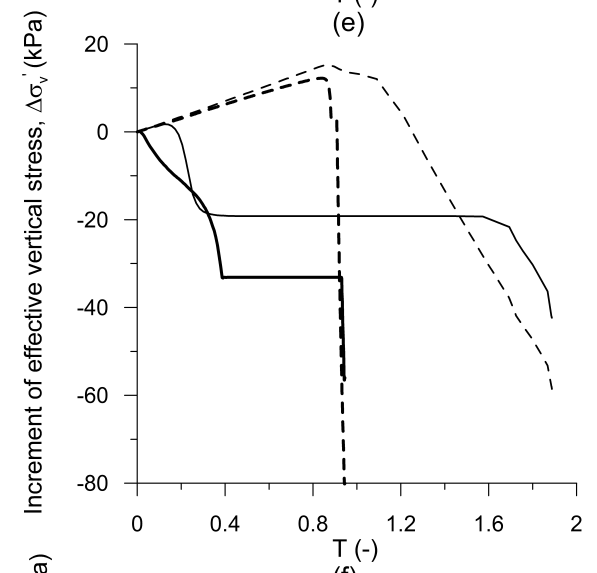

(f)

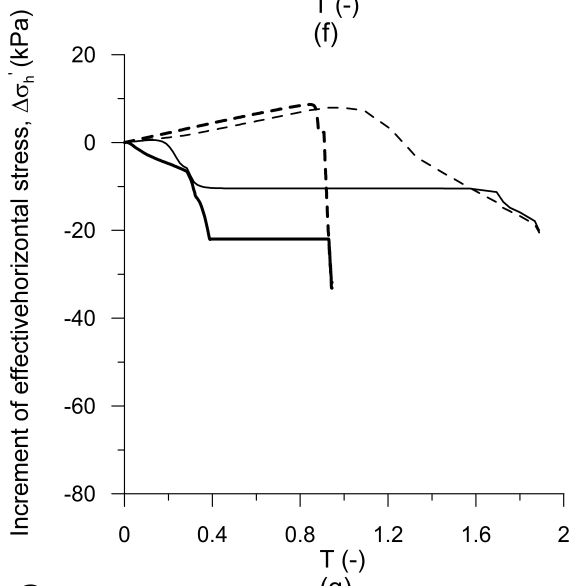

(g)

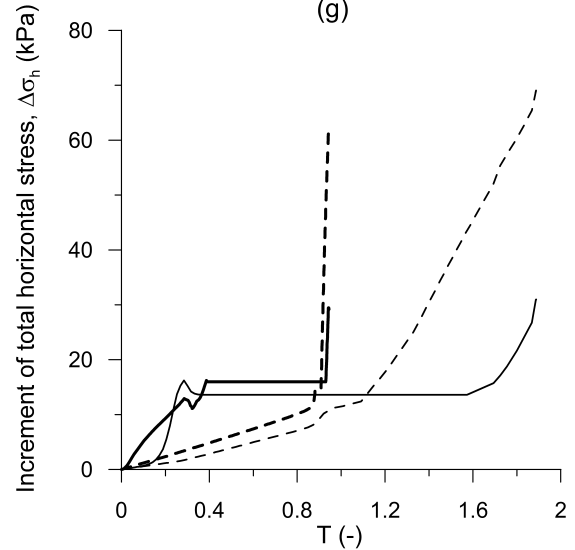

(h)

Figure 12: Time evolution of $S_{r}, p_{w}, \gamma, \varepsilon_{v}^{p}, \Delta \sigma_{v}, \Delta \sigma_{v}^{\prime}, \Delta \sigma_{h}^{\prime}, \Delta \sigma_{h}$ for flow boundary conditions. 
conditions, the increment of the gravity loading, due to the propagation of the wetting front above the reference element, forces the response in the $\left(p^{\prime}, q\right)$-plane to still move along the $K_{0}$-line (path $A B$ ). In this branch, the stress ratio remains almost constant as the variations of the saturation degree are too small for significantly affecting the size of the Cam-Clay ellipse, see [56]. This means that, going from $A$ to $B$, the yield surface expands following the plastic volumetric strain increments and therefore the response of the material, in the compressibility plane, is controlled by the NCL relative to the current saturation degree.

When the wetting front reaches the reference element, the saturation degree and the pore-water pressure start to increase and consequently the mean effective stress decreases (path $B C D$ ). Because of oedometric conditions also the deviator stress decreases along this branch. Even if the yield surface shrinks, this can be not sufficient for keeping the current stress state in the elasto-plastic regime. This occurs to the shallow element, causing its elastic swelling (see path $B C$ in Figure 11a), but not to the deep one, which abruptly suffers a volumetric collapse (see path $B D$ Figure 11b). Indeed, the reference element collapses when small suction changes are responsible for large variations of the saturation degree, say the point of maximum slope in the WRC. On the other hand, when large suction changes induce small variations of the saturation degree, the stress follows a path whose slope is univocally prescribed by the elastic coefficient $K_{0}^{e}=\nu /(1-\nu)$, see path $B C$ in Figure 11a. Going towards saturation conditions, the pronounced shrinkage of the yield surface forces the stress state of the shallow element to lie again on the current ellipse. In the elasto-plastic regime, the stress path tends towards the $K_{0}$-line and the solid skeleton collapses (path $C D$ in Figure 11a).

Finally for both elements any further increment of the saturation degree enhances a moderate shrinkage of the yield surface accompanied by stress release (path $D E$ ). Because of the pronounced unloading, associated with the abrupt increase of the pore-water pressure, stress falls into the elastic domain and, consequently, the specific volume continuously increases along the URL (path $D E F$ ).

For the analysis of the case of flow boundary conditions, the same adimensional variables as those used in the case of the surface ponding conditions were considered. Stress paths have not been depicted because they have been found to be very similar to those depicted in Figure 11 for the surface ponding conditions. This is indeed not surprising since the shapes of the stress paths are controlled by the concomitant change in suction and degree of saturation, uniquely related by the WRC, while time and velocity of front propagation are only parametrization variables of the stress paths. In the following few remarks concerning the main differences between the profiles relative to the considered boundary conditions are listed:

- An interesting outcome of the case where flow boundary conditions are prescribed relies on the fact that the degree of saturation at front advance correspond to the value for which the relative permeability is equal to 0.4 . This is indeed consistent with the imposed condition of seepage velocity $v=0.4 k_{\text {sat }}$. This implies that wetting is rather far from saturation and, consequently, the rising up of the water table takes longer time to reach the top of the layer. Coherently, the saturation degree and the pore-water pressure gradually increase and the time at which the shallow element gets saturation is definitely larger than that of the deep element. The unit soil weight, the total vertical stress increment and the plastic volumetric strain exhibit 
a two-steps behavior similar to that of the degree of saturation.

- As we already noticed, the complete saturation is due to the rising up of the water table from the bottom. As a consequence, the prescribed flow boundary condition implies the saturation front to propagate through a really partially saturated porous medium. The velocity of propagation of the front is therefore lower than that observed for surface ponding boundary conditions, where the front rises up in an almost saturated stratum.

\subsubsection{Jossigny silt}

In this section, the attention is focused on the hydro-mechanical behavior of the Jossigny silt when surface ponding or flow boundary conditions are imposed at the top of the domain. The numerical results are analysed underlining the main differences with respect to the Cervinara ash.

The time evolution of $S_{r}$ when surface ponding boundary condition is applied at the top of the silt layer is shown in Figure 10a. When the wetting front passes through the reference element, the saturation degree abruptly increases and almost saturated conditions are achieved. Comparing the responses of the two materials, a steeper variation of $S_{r}$ is observed in the Jossigny silt and the saturation is attained more quickly. The wetting front passes through the shallow elements almost at the same dimensionless time $T$, whilst it crosses the deep element of the silt before that of the ash. This means that, close to the outer surface, the imbibition is mainly driven by the saturated permeability whereas it is the dependency of relative permeability on degree of saturation that controls the hydraulic response of the deep elements.

The time evolution of pore-water pressure $p_{w}$ through the Jossigny silt does not significantly differ from that through the Cervinara soil. As already underlined for $S_{r}$, the main differences in the responses of the two materials concern with the smoother variation of $p_{w}$ in the pyroclastic soil and the time-shift in the attainment of the hydrostatic conditions.

Time evolution of the unit weight of the soil is qualitatively similar to that observed in the Cervinara soil although the initial values of $\gamma$ are significantly lower in the silt than in the pyroclastic layer. Conversely, the corresponding increments, associated with imbibition, are larger in the Cervinara than in the Jossigny soils. These features are mainly due to the differences in the initial distribution of the saturation degree and to the higher porosity of the pyroclastic soil, which can store a larger amount of water.

Regarding the behavior of the volumetric plastic strains, they are smaller in the Jossigny silt than those accumulated in the volcanic ashes. This is mainly due to the differences in the initial porosity and the parametrisation of the NCL with respect to the saturation degree.

As far as concerns the stress state, $\Delta \sigma_{v}$ increases until the saturation conditions are attained above the considered element, similarly to the pyroclastic soil. However, as saturation is achieved at different times in the two materials, $\Delta \sigma_{v}$ in the Jossigny silt approaches its stationary value when the wetting front passes through the element. Moreover, the difference in the slope of the $\Delta \sigma_{v}$ profile is justified by the larger amount of water kept inside by the pyroclastic soil.

Finally, the differences in the time evolution of $\Delta \sigma_{v}^{\prime}, \Delta \sigma_{h}^{\prime}$ and $\Delta \sigma_{h}$ for the two materials are consistent with those relative to $p_{w}$ and $\Delta \sigma_{v}$. 

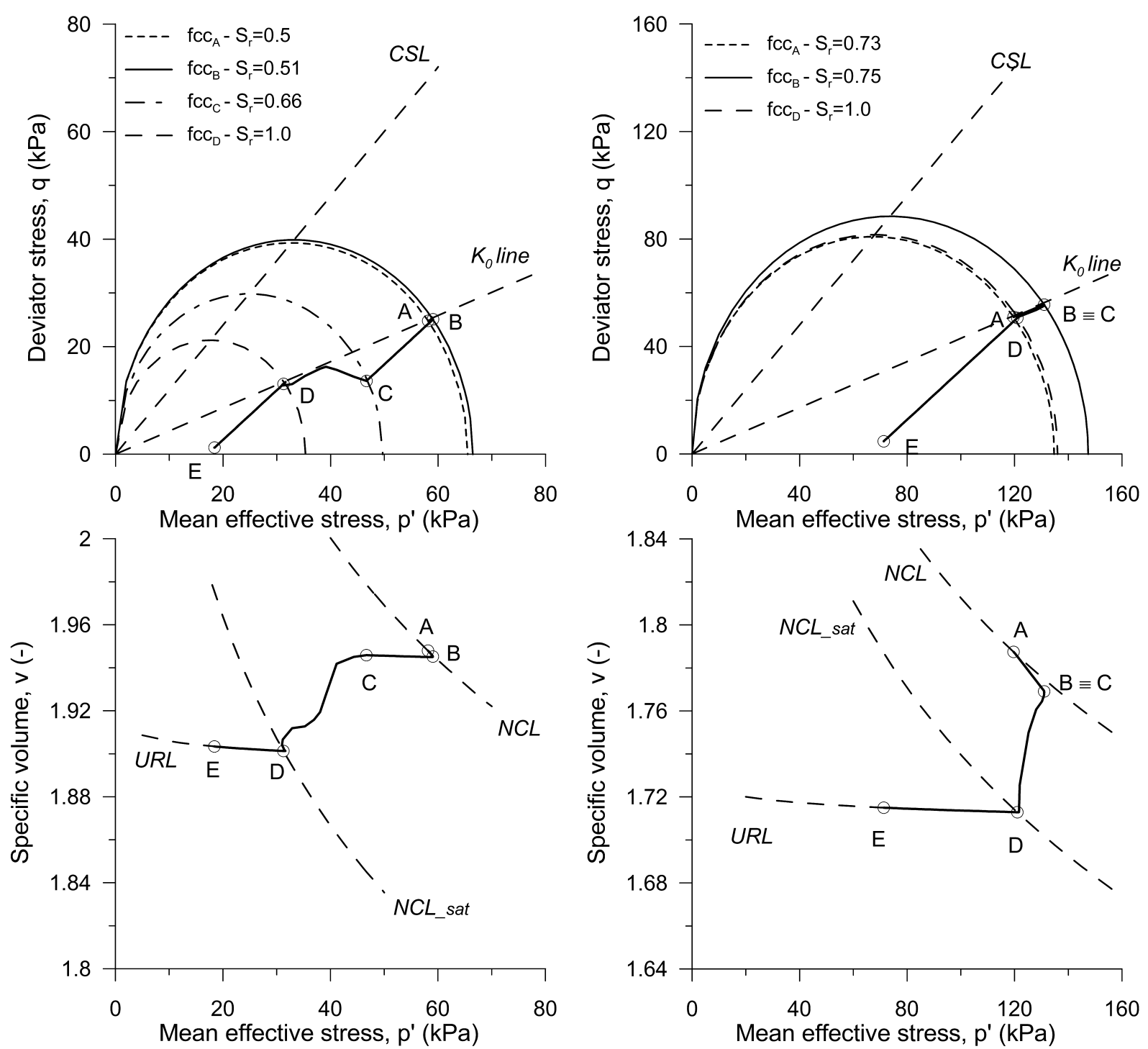

(a)

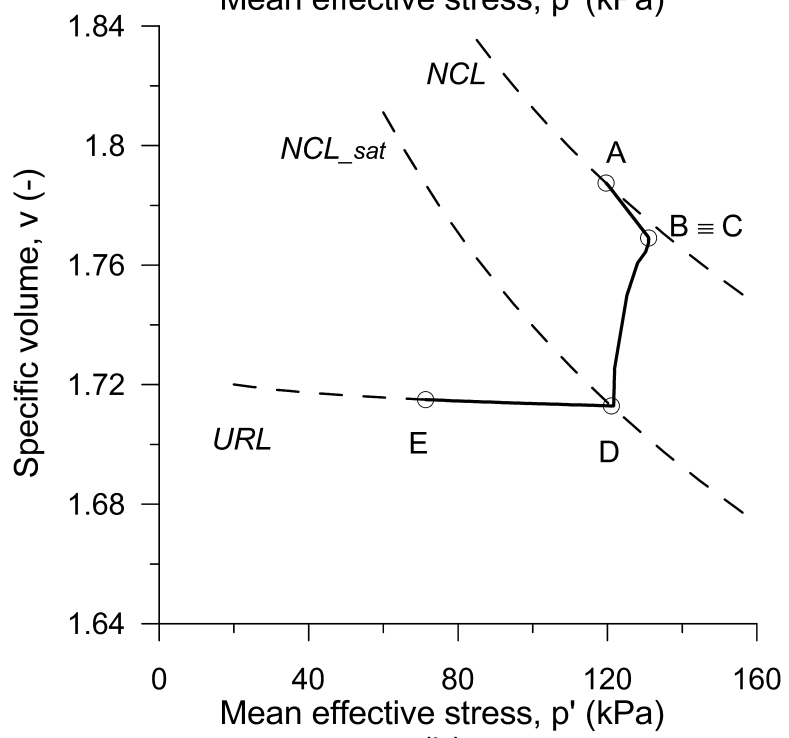

(b)

Figure 13: Stress paths and volumetric behavior for surface ponding boundary conditions for Jossigny silt: (a) shallow element: $z=2 \mathrm{~m}$; (b) deep element: $z=8 \mathrm{~m}$.

The stress paths and the volumetric behavior of the shallow and deep elements in the Jossigny soil are shown in Figure 13. The responses in the $\left(p^{\prime}, q\right)$ and $\left(p^{\prime}, v\right)$ planes do not show significant differences compared with those of the Cervinara soil. However, comparing elements at the same depth, the increment of the invariants along the path $A B$ is smaller than that observed in the Cervinara soil. This is a consequence of the lower porosity and the higher initial saturation degree of the Jossigny silt compared with the volcanic ashes. Moreover the volumetric collapse exhibited by the Jossigny silt corresponds to a negative variation of the specific volume less significant than that of Cervinara. Finally, the results obtained imposing flow boundary conditions are shown in Figure 12. As already noticed, imposing a seepage velocity equal to $0.4 k_{\text {sat }}$ corresponds to prescribe at the front a saturation degree equal to 0.72 in the volcanic ash and of 0.98 in the silt. In both cases saturation is achieved when the water table rises up through the soil layer from the bottom. However, as in the silt the water table moves upwards in an almost 
saturated stratum, the changes of $S_{r}, \gamma, \varepsilon_{v}^{p}$ and $\Delta \sigma_{v}$ due to the second part of the evolution are rather unimportant, especially for the shallow element. Similarly to the Cervinara soil, the stress paths and the volumetric behavior at shallow and deep locations are not shown because they are very similar to those presented for the case of surface ponding. It evidences again that the stress paths do not depend on the type of applied hydraulic boundary conditions.

\section{Conclusions}

In this paper we investigated the coupled hydro-mechanical response of two different partially saturated soils - a clayey silt and a volcanic ash - initially loaded by in-situ stresses and subjected to rainfall infiltration. A suitable elasto-plastic constitutive model, extended to the regime of partial saturation, has been adopted to describe the response of the soils during water infiltration in oedometric conditions. We deeply discussed the effects of the hydro-mechanical coupling in terms of the evolution of the saturation profiles and the stress paths, followed by points at different depths through the layer. The adopted constitutive model accounts for the effect of partial saturation on yielding by introducing a parametrization of the consolidation effective pressure $p_{c}^{\prime}$ on the degree of saturation. The model has been separately calibrated for the two materials using triaxial and oedometric tests under suction controlled conditions; in particular the results of equalization during suction controlled triaxial tests has been used to calibrate the unsaturated hydraulic permeability.

Concerning the hydraulic aspects of the phenomenon, saturation is mainly due to a wetting front propagating downwards, when surface ponding boundary conditions are prescribed at the top of the soil domain. Conversely, flow boundary conditions imply that the entire layer is progressively saturated only when the water table moves from the bottom to the top. Because of this discrepancy, the time necessary to saturate the entire layer is much larger when prescribing flow boundary conditions rather than surface ponding.

The hydro-mechanical coupling has also been analysed through the space-time evolution of plastic volumetric strain. The maximum of plastic compressive deformation, under surface ponding conditions, is attained at the depth of complete saturation. On the other hand, under flow boundary condition, the activation of a double mechanism of saturation, consisting into the top-down propagation of a wetting front followed by the bottom-up propagation of a saturation front, initially yields a relative maximum of the plastic strain to propagate from the top to the bottom of the layer, and later the absolute maximum to rise up from the bottom to the top. The spatial distribution of plastic deformations is particular relevant in the prediction of the settlements induced by rainfall events in shallow foundations.

Differences in hydration kinetics have an influence on the stress paths and the corresponding volumetric deformations of the soil. When the wetting front is above the target depth, the mechanical response is driven by time-varying increase of the total vertical stress, due to increase in material specific weight. Conversely, once the wetting front has passed the target depth, the mechanical response is driven by the decrease of vertical effective stress due to increases in pore-water pressure and saturation. Wetting is thus accompanied by a loading/unloading cycle in effective stress. During the latter process, 
even if the stress state points inward the yield surface, the solid skeleton could collapse because of the shrinkage of the elastic domain associated with the increment of the saturation degree.

Further developments will be devoted to extend our results to the case of non-horizontal ground surface with the aim of simulating the triggering conditions of shallow landslides induced by rainfall events and comparing it to documented case-histories.

\section{Nomenclature}

$\alpha \quad$ Hardening variable

$\beta \quad$ Force associated to the hardening variable $\alpha$

$\delta_{i j} \quad$ Kronecker delta function

$\eta_{w} \quad$ Dynamic viscosity of the wetting fluid

$\kappa \quad$ Slope of the unloading-reloading line in the semi-logarithmic compressibility plane

$\Lambda \quad$ Plastic multiplier

$\lambda \quad$ Slope of the Normal Consolidation Line in the semi-logarithmic compressibility plane

$\mathcal{V}_{f} \quad$ Velocity of the fluid material particles

$\mathcal{V}_{s} \quad$ Velocity of the skeleton material particles

$\mathcal{Z} \quad$ Locked energy

$\mathrm{M}^{w} / \mathrm{M}^{a} \quad$ Lagrangian flow vector relative to the wetting fluid/gaseous mixture

v Seapage velocity

$\phi \quad$ Lagrangian porosity

$\Phi_{s} \quad$ Dissipation relative to the solid skeleton

$\phi_{w} / \phi_{a} \quad$ Lagrangian porosity of the wetting fluid/ gaseous mixture

$\Psi_{s} \quad$ Helmholtz free energy of the solid skeleton

$\sigma_{i j}^{\prime} \quad$ Bishop stress

$\sigma_{i j} \quad$ Overall stress tensor

$\varepsilon_{i j} \quad$ Strain of the skeleton material particles

$\varkappa \quad$ Intrinsic permeability of the skeleton

$B \quad$ Width of the soil layer 


$\begin{array}{ll}b & \text { Constitutive parameter controlling the rate of change of } p_{c}^{\prime} \text { due to } S_{r} \\ e & \text { Void ratio } \\ f\left(p^{\prime} ; q ; \beta\right) & \text { Loading function } \\ f_{c c}\left(p^{\prime}, q, p_{c}^{\prime}\right) & \text { Cam-Clay yield function } \\ H & \text { Thickness of the soil layer } \\ k\left(S_{r}\right) & \text { Relative permeability of the wetting fluid } \\ K_{0} & \text { Earth pressure coefficient } \\ k_{\mathrm{sat}} & \text { Saturated permeability } \\ M & \text { Slope of the Critical State Line (CSL) } \\ m_{w} / m_{a} & \text { Fluid mass content relative to the wetting fluid/gaseous mixture } \\ n & \text { Eulerian porosity } \\ p^{\prime} & \text { Effective mean stress (positive in compression) } \\ p_{c}^{\prime} & \text { Consolidation effective pressure } \\ p_{w} / p_{a} & \text { Pressure of the wetting fluid/gaseous mixture } \\ q & \text { Deviator stress } \\ S_{r} & \text { Suction (capillary pressure) } \\ & \text { Degree of saturation of the wetting phase in the current pore space }\end{array}$

\section{References}

[1] J. Muñoz Castelblanco, P. Delage, J. Pereira, Y. Cui, et al., "Some aspects of the compression and collapse behaviour of an unsaturated natural loess," Géotechnique Letters, pp. 1-6, 2011.

[2] F. Casini, "Deformation induced by wetting: a simple model," Canadian Geotechnical Journal, vol. 49, no. 8, pp. 954-960, 2012.

[3] L. Thorel, V. Ferber, B. Caicedo, and I. Khokhar, "Physical modelling of wettinginduced collapse in embankment base," Géotechnique, vol. 61, no. 5, pp. 409-420, 2011. 
[4] O. Vilar and R. Rodrigues, "Collapse behavior of soil in a brazilian region affected by a rising water table," Canadian Geotechnical Journal, vol. 48, no. 2, pp. 226-233, 2011.

[5] E. Damiano, L. Olivares, and L. Picarelli, "Steep-slope monitoring in unsaturated pyroclastic soils," Engineering Geology, vol. 137, pp. 1-12, 2012.

[6] G. Sorbino and M. Nicotera, "Unsaturated soil mechanics in rainfall-induced flow landslides," Engineering Geology, vol. 165, pp. 105-132, 2013.

[7] J. Eichenberger, A. Ferrari, and L. Laloui, "Early warning thresholds for partially saturated slopes in volcanic ashes," Computers and Geotechnics, vol. 49, pp. 79-89, 2013.

[8] T. Zhan and C. Ng, "Analytical analysis of rainfall infiltration mechanism in unsaturated soils," International Journal of Geomechanics, vol. 4, no. 4, pp. 273-284, 2004 .

[9] R. Papa, M. Pirone, M. Nicotera, and G. Urciuoli, "Seasonal groundwater regime in an unsaturated pyroclastic slope," Géotechnique, vol. 63, no. 5, pp. 420-426, 2012.

[10] S. Springman, A. Thielen, P. Kienzler, and S. Friedel, "A long-term field study for the investigation of rainfall-induced landslides," Géotechnique, vol. 63, no. 14, pp. 11771193, 2013.

[11] E. Alonso, A. Gens, A. Josa, et al., "Constitutive model for partially saturated soils," Géotechnique, vol. 40, no. 3, pp. 405-430, 1990.

[12] S. Wheeler, "Inclusion of specific water volume within an elasto-plastic model for unsaturated soil," Geotechnical Journal, vol. 33, no. 1, pp. 42-57, 1996.

[13] H. Thomas and Y. He, "Modelling the behaviour of unsaturated soil using an elastoplastic constitutive model," Géotechnique, vol. 48, no. 5, pp. 589-603, 1998.

[14] J. Vaunat, E. Romero, and C. Jommi, "An elastoplastic hydromechanical model for unsaturated soils," in Experimental Evidence and Theoretical Approaches in Unsaturated Soils (Tarantino, A and Mancuso, C, ed.), pp. 121-138, 2000. International Workshop on Unsaturated Soils, TRENT, ITALY, APR 10-12, 2000.

[15] A. Gens, M. Sánchez, and D. Sheng, "On constitutive modelling of unsaturated soils," Acta Geotechnica, vol. 1, no. 3, pp. 137-147, 2006.

[16] D. Sheng, "Review of fundamental principles in modelling unsaturated soil behaviour," Computers and Geotechnics, vol. 38, no. 6, pp. 757-776, 2011.

[17] L. Sanavia, B. Schrefler, and P. Steinmann, "A formulation for an unsaturated porous medium undergoing large inelastic strains," Computational Mechanics, vol. 28, no. 2, pp. $137-151,2002$.

[18] A. Zhou, D. Sheng, S. Sloan, and A. Gens, "Interpretation of unsaturated soil behaviour in the stress-saturation space, i: Volume change and water retention behaviour," Computers and Geotechnics, vol. 43, pp. 178-187, 2012. 
[19] A. Zhou, D. Sheng, S. Sloan, and A. Gens, "Interpretation of unsaturated soil behaviour in the stress-saturation space: Ii: Constitutive relationships and validations," Computers and Geotechnics, vol. 43, pp. 111-123, 2012.

[20] E. Rojas and O. Chávez, "Volumetric behavior of unsaturated soils," Canadian Geotechnical Journal, vol. 50, no. 2, pp. 209-222, 2013.

[21] W. Sołowski and S. Sloan, "Equivalent stress approach in creation of elasto-plastic constitutive models for unsaturated soils," International Journal of Geomechanics, vol. 36, no. 14, pp. 1667-1681, 2012.

[22] A. Tsiampousi, L. Zdravković, and D. Potts, "A new hvorslev surface for critical state type unsaturated and saturated constitutive models," Computers and Geotechnics, vol. 48, pp. 156-166, 2013.

[23] W. Arairo, F. Prunier, I. Djéran-Maigre, and F. Darve, "A new insight into modelling the behaviour of unsaturated soils," International Journal for Numerical and Analytical Methods in Geomechanics, vol. 37, pp. 2629-2654, 2013.

[24] S. M. Lloret-Cabot M and W. SJ, "Formulation of a three-dimensional constitutive model for unsaturated soils incorporating mechanicalâĂ Şwater retention couplings," vol. 37, pp. 3008-3035, 2013.

[25] X. Song and R. Borja, "Mathematical framework for unsaturated flow in the finite deformation range," International Journal of Numerical Methods in Engineering, vol. 97, no. 9, pp. 658-682, 2014.

[26] C. Jommi, "Remarks on the constitutive modelling of unsaturated soils," in Experimental Evidence and Theoretical Approaches in Unsaturated Soils (Tarantino, A and Mancuso, C, ed.), pp. 139-153, 2000. International Workshop on Unsaturated Soils, TRENT, ITALY, APR 10-12, 2000.

[27] R. Tamagnini, "An extended cam-clay model for unsaturated soils with hydraulic hysteresis," Géotechnique, vol. 54, no. 3, pp. 223-228, 2004.

[28] E. Romero and J. Vaunat, "Retention curves of deformable clays," Experimental evidence and theoretical approaches in unsaturated soils, pp. 91-106, 2000.

[29] D. Gallipoli, S. Wheeler, and M. Karstunen, "Modelling the variation of degree of saturation in a deformable unsaturated soil.," Géotechnique., vol. 53, no. 1, pp. 105$112,2003$.

[30] A. Tarantino, "A water retention model for deformable soils," Géotechnique, vol. 59, no. 9, pp. 751-762, 2009.

[31] D. Mašín, "Predicting the dependency of a degree of saturation on void ratio and suction using effective stress principle for unsaturated soils," International journal for numerical and analytical methods in geomechanics, vol. 34, no. 1, pp. 73-90, 2010 . 
[32] F. Casini, J. Vaunat, E. Romero, and A. Desideri, "Consequences on water retention properties of double-porosity features in a compacted silt," Acta Geotechnica, vol. 7, pp. 139-150, JUN 2012.

[33] A. Tsiampousi, L. Zdravkovic, and D. Potts, "A three-dimensional hysteretic soilwater retention curve," Geotechnique, vol. 63, no. 2, pp. 155-164, 2013.

[34] S. Salager, M. Nuth, A. Ferrari, and L. Laloui, "Investigation into water retention behaviour of deformable soils," Canadian Geotechnical Journal, vol. 50, no. 2, pp. 200$208,2013$.

[35] O. Coussy, J. Pereira, and J. Vaunat, "Revisiting the thermodynamics of hardening plasticity for unsaturated soils," Computers and Geotechnics, vol. 37, no. 1, pp. 207$215,2010$.

[36] L. Picarelli, A. Evangelista, G. Rolandi, A. Paone, M. Nicotera, L. Olivares, A. Scotto di Santolo, S. Lampitiello, and M. Rolandi, "Mechanical properties of pyroclastic soils in campania region," in Invited paper, 2nd Int. Workshop on Characterisation and Engineering Properties of Natural Soils, Singapore, 2006.

[37] P. Delage, M. Audiguier, Y. Cui, and M. Howat, "Microstructure of a compacted silt," Canadian Geotechnical Journal, vol. 33, no. 1, pp. 150-158, 1996.

[38] O. Coussy, Poromechanics. Wiley. com, 2004.

[39] G. Sciarra, F. dell'Isola, N. Ianiro, and A. Madeo, "A variational deduction of second gradient poroelasticity part i: General theory," Journal of Mechanics of Materials and Structures, vol. 3, no. 3, pp. 507-526, 2008.

[40] G. Houlsby, "The work input to an unsaturated granular material," Géotechnique, vol. 47, no. 1, pp. 193-196, 1997.

[41] O. Coussy, Mechanics and physics of porous solids. John Wiley \& Sons, 2010.

[42] D. Fredlund and H. Rahardjo, Soil mechanics for unsaturated soils. John Wiley \& Sons, 1993.

[43] O. Coussy, D. P, T. Lassabatère, and V. Baroghel-Bouny, "The equivalent pore pressure and the swelling and shrinkage of cement-based materials," Materials and Structures, vol. 37, no. 1, pp. 15-20, 2004.

[44] I. Collins, "The concept of stored plastic work or frozen elastic energy in soil mechanics," Geotechnique, vol. 55, no. 5, pp. 373-382, 2005.

[45] E. Romero, G. Della Vecchia, and C. Jommi, "An insight into the water retention properties of compacted clayey soils," Géotechnique, vol. 61, no. 4, pp. 313-328, 2011.

[46] R. Tamagnini, "On the effective stress principle in unsaturated soil," Rivista Italiana di Geotecnica, vol. 3, pp. 25-31, 2011.

[47] R. Nova, Soil Mechanics. John Wiley \& Sons, 2010. 
[48] L. Olivares and L. Picarelli, "Shallow flowslides triggered by intense rainfalls on natural slopes covered by loose unsaturated pyroclastic soils," Géotechnique, vol. 53, no. 2, pp. 283-287, 2003.

[49] F. Casini, V. Serri, and S. Springman, "Hydromechanical behaviour of a silty sand from a steep slope triggered by artificial rainfall: from unsaturated to saturated conditions," Canadian Geotechnical Journal, vol. 50, no. 1, pp. 28-40, 2013.

[50] F. Casini, Effetti del grado di saturazione sul comportamento meccanico di un limo. PhD thesis, Doctoral thesis, Università di Roma La Sapienza, 2008.

[51] F. D'Onza, D. Gallipoli, S. Wheeler, F. Casini, J. Vaunat, N. Khalili, L. Laloui, C. Mancuso, D. Mašín, M. Nuth, et al., "Benchmark of constitutive models for unsaturated soils," Géotechnique, vol. 61, no. 4, pp. 283-302, 2011.

[52] J. Muñoz, V. De Gennaro, and E. Delaure, "Experimental determination of unsaturated hydraulic conductivity in compacted silt," in Unsaturated Soils. Advances in Geo-Engineering: Proceedings of the 1st European Conference, E-UNSAT 2008, Durham, United Kingdom, 2-4 July 2008, p. 123, CRC Press, 2008.

[53] R. Tamagnini, Analytical and Numerical models for unsaturated soils. PhD thesis, PhD thesis, Università di Roma âĂŸLa Sapienza, 2003.

[54] R. Borja and S. Lee, "Cam-clay plasticity, part 1: implicit integration of elasto-plastic constitutive relations," Computer Methods in Applied Mechanics and Engineering, vol. 78, no. 1, pp. 49-72, 1990.

[55] A. Burghignoli, A. Desideri, and R. Tamagnini, "Numerical modelling of dam using the modified cam clay extended to the unsaturated condition," in Third International Conference on Unsaturated Soils, vol. 1, pp. 221-225, 2002.

[56] A. Federico, G. Elia, and A. Murianni, "The at-rest earth pressure coefficient prediction using simple elasto-plastic constitutive models," Computers and Geotechnics, vol. 36, no. 1, pp. 187-198, 2009.

[57] P. Dangla and O. Coussy, "Consequences of a vanishing diffusion process in the drainage from a one dimensional sand column," in Proceedings of NAFEMS on Design, Simulation and Optimisation: Reliabiblity and Applicability of Computational Methods, 1997. 
Table 1: Hydraulic parameters for the two materials.

\begin{tabular}{cl|cc}
\hline & & Cervinara & Jossigny \\
\hline \multirow{4}{*}{ WRC } & $\alpha\left(k^{2}{ }^{-1}\right)$ & 0.13 & 0.08 \\
& $m$ & 0.57 & 0.49 \\
& $n$ & 2.32 & 1.96 \\
& $\bar{S}_{r}$ & 0.36 & 0.41 \\
\hline \multirow{3}{*}{ Permeability } & $k_{\text {sat }}(m / s)$ & $1.4410^{-6}$ & $3.6710^{-7}$ \\
& $\delta$ & 6 & 0.138 \\
& $\beta$ & 2.5 & $210^{-4}$ \\
& $n$ & 1.5 & 0.55 \\
\hline
\end{tabular}


Table 2: Mechanical parameters for the two materials.

\begin{tabular}{c|cccccc}
\hline & $\lambda$ & $\kappa$ & $M$ & $\nu$ & $b$ & $\begin{array}{c}\gamma_{s} \\
\left(k N / \mathrm{m}^{3}\right)\end{array}$ \\
\hline Cervinara & 0.22 & 0.03 & 1.45 & 0.25 & 2.3 & 25.6 \\
Jossigny & 0.14 & 0.004 & 1.2 & 0.3 & 1.97 & 26.4 \\
\hline
\end{tabular}


Table 3: Hydraulic boundary conditions: surface ponding and flow.

\begin{tabular}{c|cccc}
\hline & $z=0$ & $z=H$ & $x=0$ & $x=B$ \\
& $x \in(0, B)$ & $x \in(0, B)$ & $z \in(0, H)$ & $z \in(0, H)$ \\
\hline surface ponding & $p_{w}=0$ & $\mathrm{v}_{z}=0$ & $\mathrm{v}_{x}=0$ & $\mathrm{v}_{x}=0$ \\
flow & $\mathrm{v}_{z}=0.4 k_{\text {sat }}$ & $\mathrm{v}_{z}=0$ & $\mathrm{v}_{x}=0$ & $\mathrm{v}_{x}=0$ \\
\hline
\end{tabular}

